\title{
Scale-dependent tipping points of bacterial colonization resistance
}

\author{
Yuya Karita ${ }^{1}$, David T. Limmer ${ }^{2,3,4}$, and Oskar Hallatschek ${ }^{1, *}$ \\ ${ }^{1}$ Departments of Physics and Integrative Biology, University of California, Berkeley, California 94720 \\ ${ }^{2}$ Department of Chemistry, University of California, Berkeley, California 94720 \\ ${ }^{3}$ Kavli Energy NanoScience Institute, Berkeley, California 94720 \\ ${ }^{4}$ Chemical and Materials Science Divisions, Lawrence Berkeley National Laboratory, Berkeley, California 94720 \\ *ohallats@berkeley.edu
}

\section{ABSTRACT}

Bacteria are efficient colonizers of a wide range of secluded micro-habitats, such as soil pores ${ }^{1-3}$, skin follicles ${ }^{4}$, dental cavities $^{5,6}$ or crypts in gut-like environments ${ }^{7-9}$. Although numerous factors promoting or obstructing stable colonization have been identified ${ }^{10-14}$, we currently lack systematic approaches to explore how population stability and resilience depend on the scale of the micro-habitat. Using a microfluidic device to grow bacteria in crypt-like incubation chambers of systematically varied lengths, we found that the incubation scale can sensitively tune bacterial colonization success and resistance against invaders. Small crypts are un-colonizable, intermediately sized crypts can stably support dilute populations, while beyond a second critical lengthscale, populations phase-separate into a dilute and a jammed region. We demonstrate that the jammed state confers extreme colonization resistance, even if the resident strain is suppressed by an antibiotic. Combined with a flexible biophysical model, we show that scale acts as an environmental filter that can be tuned via the competition between growth and collective cell motion. More broadly, our observations underscore that scale can profoundly bias experimental outcomes in microbial ecology ${ }^{15-18}$. Systematic, flow-adjustable lengthscale variations may serve as a promising strategy to elucidate further scale-sensitive tipping points ${ }^{19-21}$ and to rationally modulate the stability and resilience of microbial colonizers ${ }^{22}$.

Natural microbial communities are often found to be remarkably stable, capable of either quickly recovering from disturbances or remaining essentially unaffected by them ${ }^{4,11,23,24}$. Stability is particularly puzzling in small populations, which are prone to number fluctuations and lack the size and extent to buffer against local environmental changes. Nevertheless, small but stable populations have been found in association with spatially defined micro-habitats ${ }^{4,8,12,25-28}$.

Strains that colonize cavities are sometimes found to be so stable that they hold their ground against even much fitter invaders $^{29}$. For example, Bacteroidis fragilis is a particularly resilient colonizer of crypts in the mice gut ${ }^{12}$. Conspecifics are unable to invade, unless the resident strain is strongly suppressed by an antibiotic. A similar colonization resistance has been demonstrated for groups of ceca microbiota in mice guts ${ }^{27}$ and for Lactobacillus plantarum in fly guts ${ }^{28}$.

The ubiquity of micro-habitat associated stability and colonization resistance raises the question of whether these features generically emerge in confined spaces. Previous studies have identified biological features, such as suppressed biofilm growth or the expression of specific adhesion molecules, that promote stability in specific systems ${ }^{12,13}$. However, we currently lack systematic scale-dependent measurements to identify a generic mechanism of stability and resilience in micro-habitats, as well as a theory that could predict colonization success and tipping points. To fill this gap, we developed an approach to measure the scale-dependence of microbial colonization patterns combined with a predictive theory of how microbes invade, occupy and protect confined micro-habitats.

Our experiments employ a microfluidic incubation device that allows to continuously monitor bacterial population dynamics in crypt-shaped chambers across many lengthscales (Fig. 1a). A supply channel is used to continuously perfuse the device with media enabling the experiments to run under constant conditions for several days. As bacteria are inoculated and pass through the supply channel, they get exposed to rectangular cavities of systematically varied lengths (10-350 $\mu \mathrm{m})$. Even though the fluid inside these cavities is largely stagnant, it is nutrient rich and hence supports growth, due to the rapid diffusion of small nutrient molecules from the supply channel ${ }^{30,31}$.

In this device, lengthscale-dependent ecological processes can be identified by comparing the colonization dynamics across the sequence of chambers. To capture the differential population dynamics in single microscopy frames, we ordered the cavities according to size (see Fig. ED9 for a randomized control). The device thus resembles a panflute in appearance, so we refer to our device as a "Microfluidic Panflute". We employed it to explore the colonization dynamics of several bacterial genera, focusing mainly on Acetobacter, which is prevalent in the fly gut ${ }^{28,32}$ and grows aerobically.

We found that the emerging population dynamics sensitively depend on the length of the incubation chamber. The scale 
a)

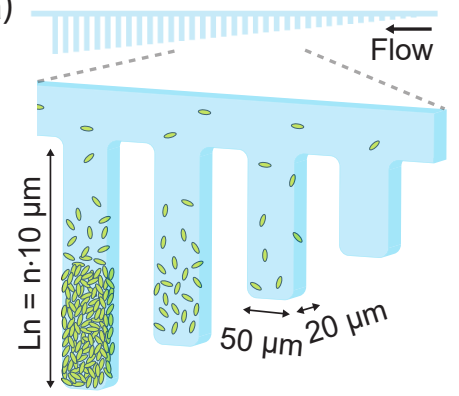

b)

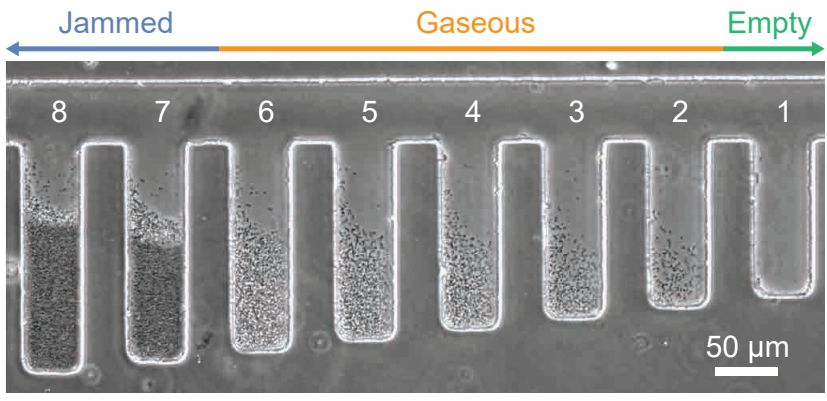

c)



d)

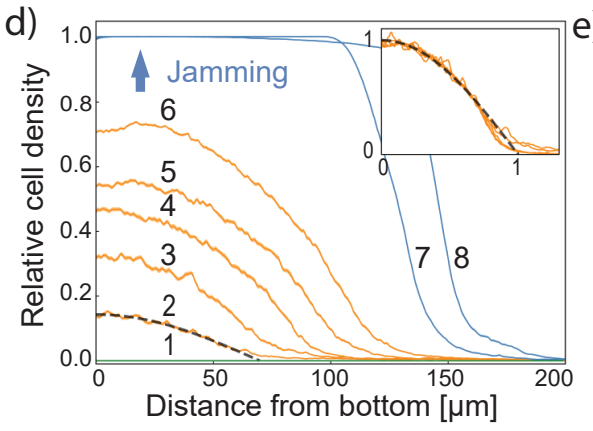

e)



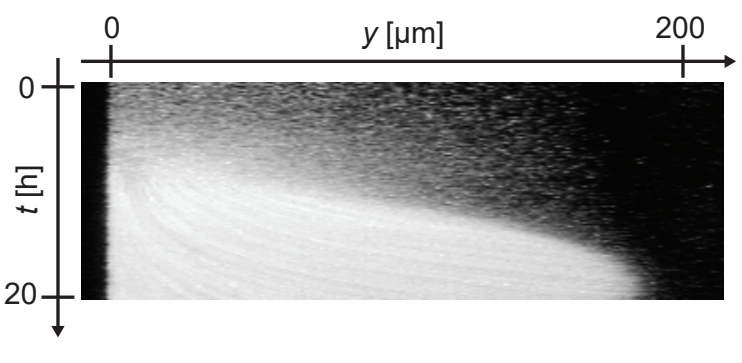

Figure 1. Microfluidic experiments reveal scale-dependent colonization patterns. (a) A scheme of our Microfluidic Panflute incubation device: Rectangular cavities of systematically varied length are connected to a common supply channel through which media and bacteria flow. (b) The steady state after five days of incubation of a fly gut bacterium (A. indonesiensis). Depending on their length, cavities could not be invaded (1), hosted gaseous population (2-6) or a phase-separated population with a jammed and gaseous population (7-8). (c) Confocal images of a partially jammed and gaseous population. (d) Steady state cell density profiles obtained from time lapse movies. The shaded regions show the standard error of the mean. The profiles of gaseous phases (orange) collapsed to our linearized establishment model (black) upon rescaling both axes (inset). (e) A kymograph of the jamming front movement.

sensitivity is particularly strong near two recognizable phase transitions:

Establishment transition. While all cavities are sporadically visited by cells, colonization attempts remain unsuccessful in small chambers. In cambers exceeding a certain threshold length, cell densities stabilize after 2-3 days of incubation and are maintained for at least five days. Cell densities, as measured from the time-averaged signal intensity, increase with chamber length, are highest at the floor of the cavities and gradually decay towards a line of zero density (Fig. 1d). We call this regime "gaseous" because the cell packing fraction is small and cells diffuse almost freely (SI Fig. ED2).

Jamming transition. When the chamber length exceeds a second threshold, a densely populated region appears at the bottom of the cavities that is sharply separated from a gaseous region towards the opening of the cavities. Confocal imaging shows that neighboring cells are in direct contact in the dense phase, which is why we call the condensed phase "jammed" (Fig. 1c). Dynamically, the jammed phase grows like a wave from the floor towards the open boundary of a chamber, as can be seen in the kymograph Fig. 1e and the SI Movie. Interestingly, the transition from gaseous to jammed is abrupt in the size of the chambers. Between two neighboring cavities, differing by just $5 \%$ in length, the colonization state transitions from gaseous to nearly $75 \%$ jammed (quantified in Fig. 1d).

We observed qualitatively similar colonization patterns for species of other genera, including $V$. cholerae and L. lactis (Fig. ED8). We therefore sought to explain the pronounced scale-sensitivity by a general species-independent mechanism.

The colonization of a cavity can be viewed as a tug of war between cell proliferation and cell removal by outflow or death ${ }^{1}$. This competition can be considered in the absence of regulation or specific cell-cell interactions, in order to discern whether the rich scale-dependent phase behavior seen in our experiments is a consequence of general biophysical processes. To describe how the cell density $c(y, t)$ at vertical position $y$ and time $t$ changes over time, we use the linear reaction-diffusion equation $\partial_{t} c(y, t)=D_{0} \partial_{y}^{2} c(y, t)+r c(y, t)$ where the first term represents cell diffusion with diffusivity $D_{0}$ and the second term represents cell proliferation with growth rate $r$. Since cells cannot penetrate the floor of the chamber, we use a reflecting boundary

\footnotetext{
${ }^{1}$ In our experiments, removal is dominated by outflow. Cell death can also be included through an effective growth rate, representing the difference between growth and death rate
} 


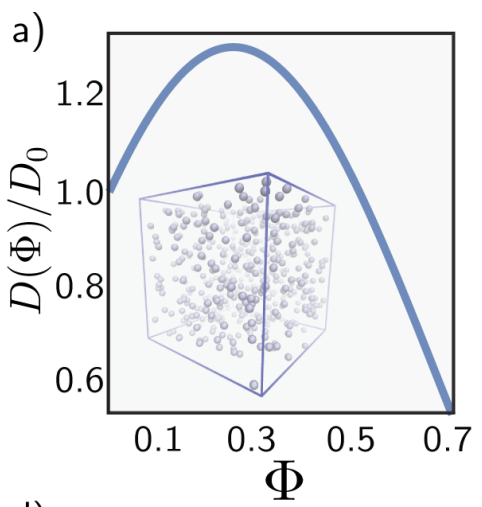

d)

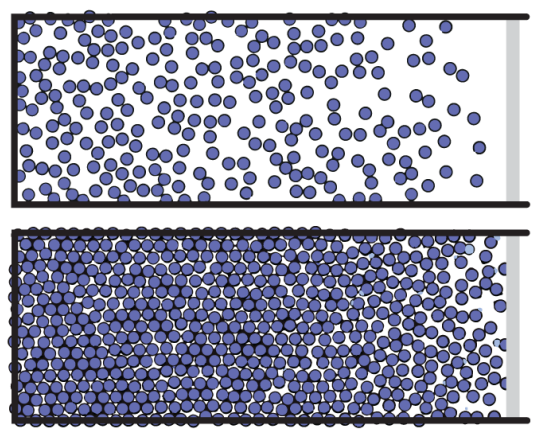

b)
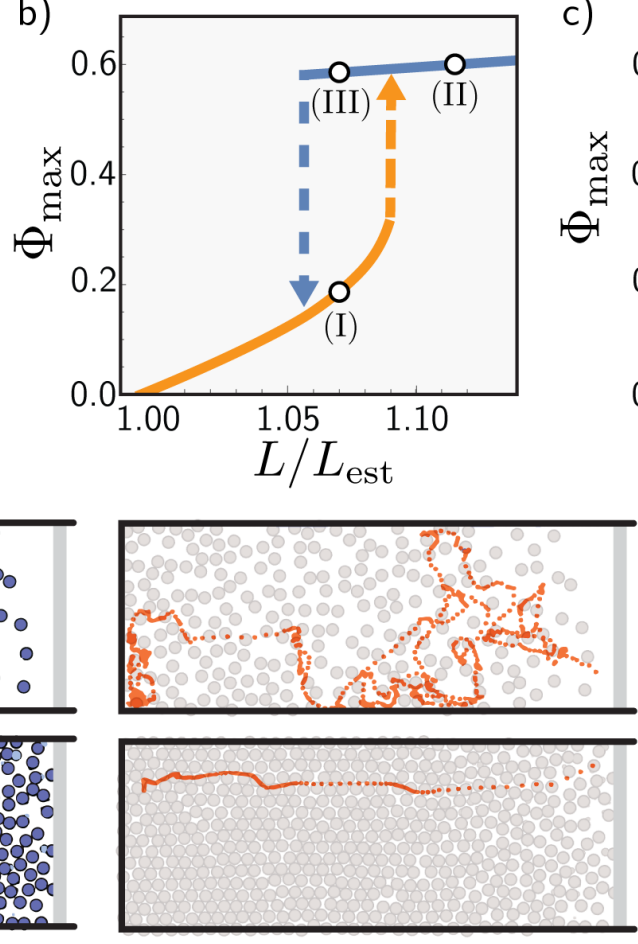

c)

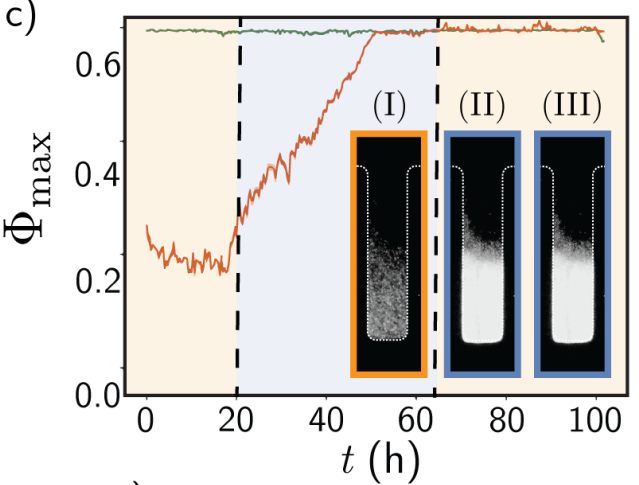

e)

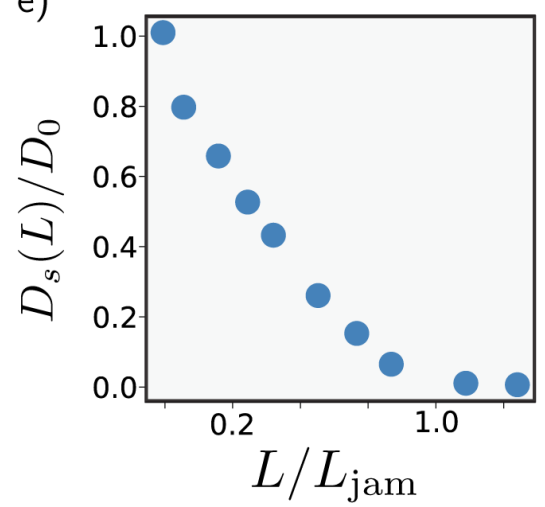

Figure 2. Theory: Collective motion can stabilize a growing population and drive phase separation. (a) Simulations show that the collective diffusivity of an idealized model of proliferating hard spheres in suspension is non-monotonic as a function of the packing fraction $\Phi=c \pi \sigma^{3} / 6$ with $\sigma$ the diameter of the particle. The negative gradients at high densities can drive a discontinuous transition towards jamming. (b) Phase diagram: The maximal packing fraction at steady state, $\Phi_{\max }$, as predicted from the density-dependent diffusivity in (a). When the control parameter $L / L_{\text {est }}$ is gradually increased, the state of the system suddenly jumps from a gaseous (I) to a partially jammed state (II, arrow pointing up). If one decreases the control parameter again, the system jumps back to a gaseous state (arrow pointing down), but at a different value of the control parameter, implying a hysteresis and a region of bistability. (c) Experiments to test bistability: A flow decrease triggers in the depicted chamber the transition from gaseous (I) to jammed (II), via an effective increase of the habitat size $L$. The orange curve depicts the density increase over time. After saturation, we increase the flow again but the chamber remains in the jammed state (III) at high density (green curve). (d) Minimal simulations of proliferating soft disks and example tagged particle trajectories for gaseous $L<L_{\text {jam }}$ (top) vs jammed states $L>L_{\text {jam }}$ (bottom) pores. (e) Self-diffusion, $D_{s}$, in the gaseous state is larger by orders of magnitude than in the jammed state, suggesting a mechanism for an invasion barrier.

condition at $y=0, \partial_{y} c(0, t)=0$. We also introduce an absorbing boundary at $y=L$ where the cells are swept away by the media flow, $c(L, t)=0$.

Our mathematical analysis (SI Text Sec. 2.1) shows that the dynamics of the density profile can be decomposed into a sum of independently evolving normal modes. The empty state is stable if the amplitude of all normal modes shrink, which requires that the scale $L$ of the population does not exceed the critical scale $L_{\text {est }}=\pi \sqrt{D_{0} / r} / 2$. In turn, this implies that bacteria can establish in a chamber only if $L>L_{\mathrm{est}}$. Thus, establishment is promoted by increasing the growth rate or decreasing the diffusivity, which drives the cell leakage. Using the measured growth rate, $r \approx 0.33 \pm 0.01 \mathrm{~h}^{-1}$ (Fig. ED1a), and diffusivity, $D_{0} \approx 0.37 \times 10^{3} \pm 0.01 \mu \mathrm{m}^{2} / \mathrm{h}$ (Fig. ED2), we estimate establishment in our experiments to occur at the scale $L_{\text {est }} \approx 53 \pm 1$ $\mu \mathrm{m}$. This is consistent with the empirical value $53 \pm 7 \mu \mathrm{m}$ that we extrapolate from our measurements (Fig. ED3b). We also confirmed that the establishment length changes predictably with variations in growth rate (Fig. ED4). More importantly, the measured density profiles agree well with the cosine shape of the first normal mode, as observed in Fig. 1d, which is expected to dominate close to the onset of colonization (SI Text 2.2). Our analysis is best suited to describe the bulk of the population where cell motion is dominated by diffusion. Deviations are expected, and indeed visible around the opening of cavities (near vanishing cell density) where the flow of the media is not negligible.

Our linear model can tell us whether bacteria grow in empty chambers but it remains blind to how a population of successful 
colonizers reaches a steady state with a finite population size and how stable this state is. To predict the long-term dynamics, we needed to include a (non-linear) population control term that modulates the competition between cell proliferation and removal. For example, bacterial batch cultures are often limited by nutrient deprivation or waste product accumulation, implying that the growth rate is not constant but decays with density (logistic population control). However, growth rates in the jammed and dilute phase were statistically indistinguishable (SI Sec. 1.8), suggesting that nutrient deprivation did not limit population growth. Therefore, we hypothesized that, while the growth rate remains approximately constant, the population outflow adjusts itself via a density-dependent diffusivity $D(c)$. Steady state is reached when the cell leakage matches the influx of newborn cells in the bulk of the chamber.

Our mathematical analysis shows that a monotonically increasing $D(c)$ (more cells $\rightarrow$ more outflow) is capable of stabilizing a gaseous state inside the chambers (SI Sec. 2.2). However, to reproduce a sudden jamming transition, $D(c)$ has to have a region of negative slope at high densities (more cells $\rightarrow$ less outflow). Intuitively, this generates a positive feedback cycle. As the density fluctuates up, diffusion-induced outflow goes down, which leads to even higher cell densities, suppressing outflow even more and so on. The cycle only breaks when the bacteria jam and come into contact, upon which the bulk modulus and, hence, $D(c)$ shoot up by several orders of magnitude ${ }^{33}$.

The required negative slope of $D(c)$ could be induced at high density by constitutive or crowding-induced stickiness between cells, or active motility, which has been shown to drive phase-separation ${ }^{34}$. Our simulations (Fig. 2) and analytical arguments (SI Sec. 2.5) show that even purely repulsive, spherical, non-motile particles exhibit a qualitatively similar phase behavior as seen our experiments. Thus, a transition between gaseous and partially jammed states emerges without any special biotic factors other than proliferation.

Our theory predicts that the jamming transition arises through a fold bifurcation and, therefore, should have the characteristics of a tipping point ${ }^{19-21}$. In particular, once a chamber becomes jammed it is not easily unjammed and requires a substantial perturbation of the control parameters (growth rate or diffusivity). This also implies that there must be region of bistability, where in the same chamber two states are stable - one gaseous and one phase-separated state (Fig. 2b). We confirmed that, in our experiments, chambers near the jamming transition indeed show bistability (Fig. 2c) by flipping from one state to another using flow modulation (Methods).

Simulations of a proliferating soft sphere model (see SI for details), further show that the cellular self-diffusion is dramatically reduced upon jamming, consistent with an onset of rigidity, except for movement of order one cell diameter per doubling induced by the division process (Fig. 2d). While in our experiments we could not track single cells in the jammed phase, we could track lineages using fluorescent tracers (Fig. ED6), which also suggests self-diffusion to drop by 2 orders of magnitude from the gaseous to the jammed state.

A drop in self-diffusion has important consequences for species invasions. It lowers the chance for outside cells to diffusively penetrate the jammed fraction against the proliferation current coming from the floor of the chamber. Accounting for this crowding-induced diffusion barrier in a theory of strain invasion (SI Sec. 4.1), we predict that the rate at which an external strain invades a jammed resident population is exponentially small in the ratio of the thickness of the jammed phase and the cell diameter. Thus, invasion of jammed populations should be an extremely rare event.

To test this prediction, we performed specific invasion experiments. We inoculated our device with the wild type strain of A. indonesiensis and waited until a steady state was reached. We then flowed in a sister strain of the same species, which is fluorescently labeled green and resistant to the drug tetracycline. Titration of tetracycline then allowed us to tune the growth rate advantage of the invading strain.

In the absence of antibiotics, we did not observe any successful invasion over experimental time scale (five days). When we added $10 \mu \mathrm{g} / \mathrm{ml}$ of the antibiotic (60\% of MIC), a scale-dependent invasion dynamics ensued: In the initial 24 hours, the drug-sensitive populations decreased the population density, thus shifting the phase boundary between gaseous and jammed to larger cavities. Over the next 48 hours, drug resistant cells entered and seized a substantial number of the gaseous chambers (Fig. 3c). Upon successful invasion, the population density generally increased again. Importantly, while most of the gaseous chambers were ultimately invaded, none of the jammed chambers did (out of 7 colonized panflutes monitored over 2-5 days in 3 independent experiments). The primary effect of the antibiotic is to push the state of some of the chambers from jammed to gaseous, upon which invasion becomes possible (Fig. 3a). Thus, while crowding strongly protects jammed populations from invasion, residents can be dislodged nevertheless if they are driven past a tipping point into a more fragile (gaseous) ecological state.

In sum, we have shown that in spatially restricted cavities, microbial populations can exhibit a rich spectrum of scaledependent colonization patterns. The physical structure of the micro-environment acts an ecological filter, permitting stable and resilient colonization only by species with matching traits. By modulating the physical characteristics of this filter, hosts may actively or passively shape the pool of potential bacterial residents, which possibly contributes to a substantial, unexplained, individuality in some host-associated microbial communities ${ }^{27}$. Conversely, species like A. indonesiensis might have adapted to this ecological filter to improve colonization rates, for instance by tuning their shape, motility, cell-surface or cell-cell 


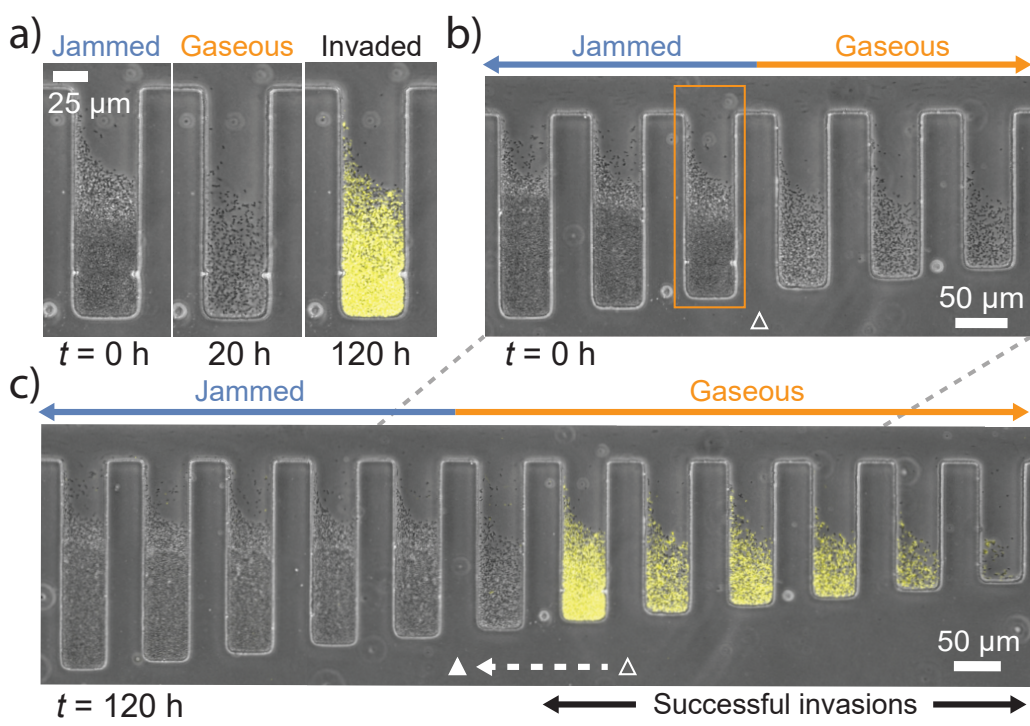

Figure 3. Crowding induced colonization resistance. (a) A schematic of our invasion experiments. After the chambers were pre-colonized by the wild type strain (dark), we introduced fluorescently labelled 'invader' strain (yellow). To make invasions more likely, we also increased the fitness of the invader by the simultaneous injection of an antibiotic (tetracycline) to which the invader was made resistant against. (b) A steady state of sensitive populations before the invaders were inoculated. The unfilled triangle shows the transition point between jammed and gaseous phases. (c) Population decay of drug-sensitive cells (dark) led to a phase change (jammed $\rightarrow$ gaseous), upon which the tetracycline-resistant strain successfully invaded. (d) 120 hours after tetracycline was added to the culture medium. Drug-sensitive populations (dark) that remained jammed were not invaded. The unfilled and filled triangle show the transition points between jammed and gaseous phases at $t=0 \mathrm{~h}$ and $t=120 \mathrm{~h}$ respectively. The injection of the growth inhibitor (tetracycline) shifted the transition point.

interactions ${ }^{35}$.

While the colonization patterns observed in our experiments could be explained by density-dependent collective diffusion alone, we expect them to be generally influenced by other biotic and abiotic factors. For example, crowding will be promoted if cells stick to one another directly or indirectly through biofilm formation, or if nutrients are supplied from the floor of the chamber. On the other hand, both establishment and jamming tends to be hindered by strong nutrient limitations or bacterial motility. While further research is needed to explore the relative importance of these factors, their impact may be anticipated theoretically using a reaction-diffusion model, which entails a flexible approach to analyze steady states (SI Sec. 2.3).

More broadly, our results underscore that the lengthscale of experimentation can have a strong influence on micro-ecological processes, which could confound experiments that do not control for scale variation ${ }^{15}$ - a well appreciated problem in the macro-ecological context ${ }^{17,18}$. Flow-tunable scale variations as implemented in our Microfluidics Panflute offer a systematic experimental approach to detect or exclude scale sensitivity in a wide range of culturable microbial communities. Since the time scales of microbial evolution and ecology are inter-twined, we expect such scale sensitive experiments an exciting avenue for future eco-evolutionary research ${ }^{36}$.

\section{Acknowledgments}

We would like to thank all members of the Hallatschek lab for helpful discussions. We also thank Arolyn Conwill and Tami Lieberman for their insightful and helpful comments. The Acetobacter strains are generous gifts by Will Luddington, whom we also thank for useful discussions at the outset of the project. Research reported in this publication was supported by the National Institute of General Medical Sciences of the National Institutes of Health under award R01GM115851, a National Science Foundation CAREER Award (1555330) and a Simons Investigator award from the Simons Foundation (327934). DTL was supported by NSF Grant CHE-195458.

\section{Author contributions statement}




\section{References}

1. Ranjard, L. \& Richaume, A. Quantitative and qualitative microscale distribution of bacteria in soil. Res. microbiology 152, $707-716$ (2001).

2. Torsvik, V. \& Øvreås, L. Microbial diversity and function in soil: from genes to ecosystems. Curr. opinion microbiology $\mathbf{5}$, 240-245 (2002).

3. Grundmann, G. L. Spatial scales of soil bacterial diversity-the size of a clone. FEMS microbiology ecology 48, 119-127 (2004).

4. Oh, J. et al. Temporal stability of the human skin microbiome. Cell 165, 854-866 (2016).

5. Proctor, D. M. \& Relman, D. A. The landscape ecology and microbiota of the human nose, mouth, and throat. Cell host \& microbe 21, 421-432 (2017).

6. Mark Welch, J. L., Dewhirst, F. E. \& Borisy, G. G. Biogeography of the oral microbiome: the site-specialist hypothesis. Annu. review microbiology 73, 335-358 (2019).

7. Tropini, C., Earle, K. A., Huang, K. C. \& Sonnenburg, J. L. The gut microbiome: connecting spatial organization to function. Cell host \& microbe 21, 433-442 (2017).

8. Donaldson, G. P., Lee, S. M. \& Mazmanian, S. K. Gut biogeography of the bacterial microbiota. Nat. Rev. Microbiol. 14, 20-32 (2016).

9. Mowat, A. M. \& Agace, W. W. Regional specialization within the intestinal immune system. Nat. Rev. Immunol. 14, 667-685 (2014).

10. Or, D., Smets, B. F., Wraith, J., Dechesne, A. \& Friedman, S. Physical constraints affecting bacterial habitats and activity in unsaturated porous media-a review. Adv. Water Resour. 30, 1505-1527 (2007).

11. Griffiths, B. S. \& Philippot, L. Insights into the resistance and resilience of the soil microbial community. FEMS microbiology reviews 37, 112-129 (2013).

12. Lee, S. M. et al. Bacterial colonization factors control specificity and stability of the gut microbiota. Nature 501, 426-429 (2013).

13. Coyte, K. Z., Tabuteau, H., Gaffney, E. A., Foster, K. R. \& Durham, W. M. Microbial competition in porous environments can select against rapid biofilm growth. Proc. Natl. Acad. Sci. 114, E161-E170 (2017).

14. Yang, C. \& Ottemann, K. M. Control of bacterial colonization in the glands and crypts. Curr. opinion microbiology 47, 38-44 (2019).

15. Ladau, J. \& Eloe-Fadrosh, E. A. Spatial, temporal, and phylogenetic scales of microbial ecology. Trends microbiology 27, 662-669 (2019).

16. Ross, S. R.-J. et al. Illuminating the intrinsic and extrinsic drivers of ecological stability across scales. Ecol. Res. (2021).

17. Wiens, J. A. Spatial scaling in ecology. Funct. ecology 3, 385-397 (1989).

18. Levin, S. A. The problem of pattern and scale in ecology: the robert h. macarthur award lecture. Ecology 73, 1943-1967 (1992).

19. Wissel, C. A universal law of the characteristic return time near thresholds. Oecologia 65, 101-107 (1984).

20. Van Nes, E. H. \& Scheffer, M. Slow recovery from perturbations as a generic indicator of a nearby catastrophic shift. The Am. Nat. 169, 738-747 (2007).

21. Dai, L., Vorselen, D., Korolev, K. S. \& Gore, J. Generic indicators for loss of resilience before a tipping point leading to population collapse. Science 336, 1175-1177 (2012).

22. Walter, J., Maldonado-Gómez, M. X. \& Martínez, I. To engraft or not to engraft: an ecological framework for gut microbiome modulation with live microbes. Curr. opinion biotechnology 49, 129-139 (2018).

23. Lozupone, C. A., Stombaugh, J. I., Gordon, J. I., Jansson, J. K. \& Knight, R. Diversity, stability and resilience of the human gut microbiota. Nature 489, 220-230 (2012).

24. Coyte, K. Z., Schluter, J. \& Foster, K. R. The ecology of the microbiome: networks, competition, and stability. Science 350, 663-666 (2015).

25. Pédron, T. et al. A crypt-specific core microbiota resides in the mouse colon. MBio 3, e00116-12 (2012). 
26. Saffarian, A. et al. Crypt-and mucosa-associated core microbiotas in humans and their alteration in colon cancer patients. MBio 10 (2019).

27. Martínez, I. et al. Experimental evaluation of the importance of colonization history in early-life gut microbiota assembly. Elife 7, e36521 (2018).

28. Obadia, B. et al. Probabilistic invasion underlies natural gut microbiome stability. Curr. Biol. 27, 1999-2006 (2017).

29. Lawley, T. D. \& Walker, A. W. Intestinal colonization resistance. Immunology 138, 1-11 (2013).

30. Wang, P. et al. Robust growth of escherichia coli. Curr. biology 20, 1099-1103 (2010).

31. Lambert, G. \& Kussell, E. Memory and fitness optimization of bacteria under fluctuating environments. PLoS Genet. 10, e1004556 (2014).

32. Yamada, R., Deshpande, S. A., Bruce, K. D., Mak, E. M. \& William, W. J. Microbes promote amino acid harvest to rescue undernutrition in drosophila. Cell reports 10, 865-872 (2015).

33. Chu, E. K., Kilic, O., Cho, H., Groisman, A. \& Levchenko, A. Self-induced mechanical stress can trigger biofilm formation in uropathogenic escherichia coli. Nat. communications 9, 1-10 (2018).

34. Cates, M. E. \& Tailleur, J. Motility-induced phase separation. Annu. Rev. Condens. Matter Phys. 6, $219-244$ (2015).

35. Kamada, N., Chen, G. Y., Inohara, N. \& Núñez, G. Control of pathogens and pathobionts by the gut microbiota. Nat. immunology 14, 685-690 (2013).

36. Dakos, V. et al. Ecosystem tipping points in an evolving world. Nat. ecology \& evolution 3, 355-362 (2019).

37. Savitzky, A. \& Golay, M. J. Smoothing and differentiation of data by simplified least squares procedures. Anal. chemistry 36, 1627-1639 (1964). 


\section{Methods}

\subsection{Bacterial strains and culture condition}

The Acetobacter indonesiensis strains were derived from SB003 (kindly gifted by William Ludington, Carnegie Institution for Science), which was originally isolated from lab flies (D. melanogaster $)^{28,32}$. SB003 was transformed with mGFP5 via the backbone plasmid pCM62 $2^{40}$ by Benjamin Obadia (not yet published). For culturing, all strains were grown in MRS medium (BD) at $30^{\circ} \mathrm{C}$. Strains are selected with $15 \mu \mathrm{g} / \mathrm{mL}$ tetracycline (Corning Cellgro) if needed.

\subsection{Microfluidics fabrication}

The microfluidic devices were fabricated by soft lithography ${ }^{41}$. In order to make a master mold, a 20 $\mu$ m-thick layer of negative photoresist (SU8-2010, MicroChem) was spin-coated on a silicon wafer (WaferNet) and patterned by photolithography with a mask aligner (Hybralign 200, OAI) through a photomask (CAD/Art Services). On the master mold, Polydimethylsiloxane (PDMS, Sylgard 184, Dow Corning) was poured with crosslinker at 10:1 ratio and cured at $60{ }^{\circ} \mathrm{C}$ in an oven overnight. The patterned PDMS was punched to make holes for inlets and outlets. The PDMS was bonded to a glass coverslip with $\mathrm{O}_{2}$ plasma treatment by a reactive ion etcher (Plasma Equipment Technical Services).

\subsection{Microfluidic cell culture}

Prior to microfluidic culture, cells were streaked on a plate from frozen stock and grown in a test tube with $3 \mathrm{~mL}$ MRS for $1-2$ days. The suspension of cells was injected into the microlfuidic device and cultured for 3-5 days with a continuous supply of the fresh medium until the system reaches a steady state. The temperature was regulated at $30{ }^{\circ} \mathrm{C}$ by a microscope incubator (H201-T and UNO, Okolab), and the flow rate of the culture medium was controlled at $4 \mu \mathrm{L} / \mathrm{h}$ by syringe pumps (neMESYS, CETONI). Images were taken by inverted microscopes (IX81, Olympus, and Eclipse Ti, Nikon).

\subsection{Density profile measurement}

To quantitatively measure the density profile of cellular populations in microfluidic crypts, GFP-tagged cells were cultured in the microfluidic panflute device for about three days. After the system reached a steady state, fluorescent intensities were measured every 20 minutes for two days. The intensities were averaged over the time points at each position and scaled by the normalization factor so that the density of jammed populations was defined as 1.

\subsection{Neutral competition and invasion with fitness effect}

To observe competitions of two strains with and without fitness effects, wild-type and GFP-tagged strains were co-cultured. As the GFP-tagged strain was resistant to tetracycline, with $10 \mu \mathrm{g} / \mathrm{mL}$ of tetracycline, the GFP-tagged cells grew normally while the wild-type strain stopped to grow. We confirmed that there was no significant growth rate difference between the strains in the absence of antibiotics.

For neutral competition experiments, a 50:50 mixture of dark and GFP-tagged cells were inoculated into the microfluidic panflute device and cultured for two days. As each type of cells colonized chambers stochastically, we parallelized six rows of the panflutes and selected chambers with a nice initial population ratio. The population dynamics were observed with a fluorescent microscope every 20 minutes for a day.

To test the colonization resistance of jammed populations, we first cultured wild-type cells in the microfluidic device. After the populations reach a steady state, the culture medium was changed from MRS to MRS $+10 \mu \mathrm{g} / \mathrm{mL}$ tetracycline, and GFP-tagged cells were continuously flowed into the device. The resulting population decay and invasion dynamics were observed with a microscope every 20 minutes for two days. In addition, the snapshots of the populations were taken every day for five days.

\subsection{Flow and temperature change experiments}

To investigate the effect of the system's parameters on the population density in microfluidic crypts, we dynamically changed the flow rate to tune the effective chamber depth. We initially cultured cells at $10 \mu \mathrm{L} / \mathrm{h}$ flow rate for three days until the system reached a steady state, and changed the flow rate to $4 \mu \mathrm{L} / \mathrm{h}$. The decrease of the flow rate affected how deep the streamlines invaded chambers and changed the effective chamber depth by 5-10 $\mu \mathrm{m}$. After the system reached a second steady state, we recovered the flow rate to $10 \mu \mathrm{L} / \mathrm{h}$ to investigate hysteresis.

We also dynamically changed the temperature of the incubation chamber for controlling the growth rate of cells. We first cultured cells at $22{ }^{\circ} \mathrm{C}$, where the growth rate is $0.281 / \mathrm{h}$, until a steady state, and ramped up the temperature to $30{ }^{\circ} \mathrm{C}$, where the growth rate is $0.331 / \mathrm{h}$ (for the measurement of the growth rate, see SI). After a second steady state, we recovered the temperature to $22{ }^{\circ} \mathrm{C}$ to see hysteresis. The transition dynamics were recorded every 20 minutes with a microscope. 
bioRxiv preprint doi: https://doi.org/10.1101/2021.05.13.444017; this version posted May 15, 2021. The copyright holder for this preprint (which was not certified by peer review) is the author/funder, who has granted bioRxiv a license to display the preprint in perpetuity. It is made available under aCC-BY-NC 4.0 International license.

\subsection{Colonization experiments with other species}

Colonization dynamics in a microfluidic panflute were tested with various microbial species (Escherichia coil, Bacillus subtilis, Vibrio cholerae, Acetobacter pasteurianus, Acetobacter tropicalis, and Lactococcus lactis. See Tabel 1 for the strain details and culture media.). Cells were streaked on a plate from frozen stock, and a small number of cells from a single colony were grown in a test tube with $3 \mathrm{~mL}$ of a culture medium for $1-2$ days (at $37^{\circ} \mathrm{C}$ for Escherichia coil and $30^{\circ} \mathrm{C}$ for the other species). The cell suspension was injected into a microlfuidic panflute and cultured for 2-6 days with a continuous supply of fresh media until the system reaches a steady state. During the microfluidic culture, the temperature was regulated at $30^{\circ} \mathrm{C}$ for all species.

\subsection{Growth rate measurement}

The growth rate of cells was measured in two ways: growth assay with a plate reader and particle image velocimetry (PIV) of a jammed population on microfluidics. Prior to the measurements, cells were cultured in test tubes from single colonies for 1-2 days in MRS at $30{ }^{\circ} \mathrm{C}$ up to saturation. For the plate reader experiments, cell suspensions were diluted to 0.02 OD, and $200 \mu \mathrm{L}$ of the suspensions were transferred to transparent flat-bottom 96-well plates (Thermo Fisher Scientific). The plates were incubated in a plate reader (Spectramax) at $30^{\circ} \mathrm{C}$, and the optical density (OD) was measured at $600 \mathrm{~nm}$ wavelength every 5 minutes with 30-second mixing before each measurement. The maximum growth rate was calculated by fitting an exponential curve to the initial 2-hour growth. The growth rate of Acetobacter indonesiensis was measured as $0.325 \pm .003$ [1/h] (Fig ED1a).

For the PIV measurement on microfluidics, cells were injected into a microfluidic device and incubated in a table-top incubator until cells colonized chambers and formed stable populations. Bright-field images were taken every 3 minutes for 3 hours and analyzed with PIVlab in Matlab ${ }^{42}$. PIV calculated the displacements of cells per timeframe. The displacement of a cell at position $y=y_{0}$ was caused by the growth of cells at $y \in\left[0, y_{0}\right]$, and therefore, the displacement at position $y=y_{0}$ could be formulated as $d\left(y_{0}\right)=y_{0}\left(e^{r \Delta t}-1\right)$. Since our timeframe ( $\Delta t=3$ minutes) was much smaller than the doubling time of the cell (2.1 hours), it held $d\left(y_{0}\right) / \Delta t \approx r y_{0}$. Thus, the slope of the velocity field in the y-direction gave the growth rate. The growth rate of Acetobacter indonesiensis was measured as $0.332 \pm .007$ [1/h] (Fig ED1d).

\subsection{Diffusivity measurements}

\subsubsection{Self-diffusivity.}

To estimate the self-diffusivity of cells in gaseous and jammed phases, the displacement of cells was tracked over time, and the mean squre displacements were calculated. A 50:50 mixture of dark and gfp-tagged cells was injected in a microfluidic panflute and cultured until the system reached a steady state. The motions of gfp-tagged cells were recorded with a fluorescent microscope every 30 seconds for 10 minutes for gaseous phases and every 20 minutes for 20 hours for jammed phases. The displacement of cells in gaseous phases was automatically tracked with TrackMate in Fiji ${ }^{43}$, and that in jammed phases was manually tracked with the Manual Tracking plugin of ImageJ.

\subsubsection{Collective Diffusivity.}

To determine the collective diffusivity, we adapted the Boltzmann-Matano analysis ${ }^{44}$ to the present case of a reaction-diffusion system. Under the assumption that our general reaction-diffusion model, $\partial_{t} c(y, t)=\partial_{y}\left[D(c(y, t)) \partial_{y} c(y, t)\right]+r c(y, t)$, is valid, we can express the density-dependent diffusivity $D(c)$ in terms of the steady state density profile as follows:

$$
D(c(y))=\frac{r \int_{0}^{y} c\left(y^{\prime}\right) d y^{\prime}}{-\partial_{y} c(y)}
$$

This equation allows us to estimate $D(c)$ from the exponential growth rate (measured as in Sec. 1.8) and the steady state density profiles. The steady-state density profiles are determined from time averaging the signal intensity of fluorescent time lapse movies. For the data in Fig. ED2c, we averaged the density profile over 20 frames (7 hours) and locally approximated it with a quadratic function by the Savitzky-Golay method ${ }^{37}$ to extract $\partial_{y} c(y)$. We excluded the y-region $20 \%$ from the opening where the flow impacted the tail of the density profile, and excluded the y-region $20 \%$ from the bottom where $\left(\partial_{y} c(y)\right)^{-1}$ was diverging. 


\section{Supplementary Information}

\section{Theory of Colonization}

In order to understand the relaxation and steady-state properties of the bacterial populations in the variable length cavities, we have considered a general reaction diffusion equation. Specifically, assuming the concentration profile is uniform along the direction perpendicular to the chamber opening, we can consider a continuity equation along just the $y$ direction. In terms of the packing fraction, $\Phi(y, t)$ at position $y$ and time $t$, (which is the concentration multiplied by the cell volume) a general reaction diffusion equation reads

$$
\partial_{t} \Phi(y, t)=\partial_{y} D[\Phi(y, t)] \partial_{y} \Phi(y, t)+b[\Phi(y, t)]
$$

where $D[\Phi(y, t)]$ is a packing fraction dependent diffusivity and $b[\Phi(y, t)]$ is a nonlinear growth term. In what follows, we will consider specific limiting cases of this general equation in order to make specific statements regarding the stability and dynamics of colonization.

\subsection{Linear Stability Analysis}

In order to understand the initial establishment of the population, we consider the linear stability of the cell density in the low density limit with constant diffusivity $D_{0}$ and growth rate $r$. This allows us to accurately determine $L_{\text {est }}$. We first expand the cell density as

$$
\Phi(y, t)=\sum_{n=0}^{\infty} a_{n}(t) \varphi_{n}(y)
$$

in terms of cosine modes

$$
\varphi_{n}(y)=\cos \left(k_{n} y\right) \quad k_{n}=\left(n+\frac{1}{2}\right) \frac{\pi}{L}
$$

which are orthonormal

$$
\left\langle\varphi_{n} \mid \varphi_{m}\right\rangle=\delta_{n m}
$$

with respect to the scalar product

$$
\langle f \mid g\rangle \equiv \frac{2}{L} \int_{0}^{L} d y f(y) g(y) .
$$

In terms of mode amplitudes $a_{n}(t)$, the linearized reaction-diffusion equation $\partial_{t} \Phi=D_{0} \partial_{y}^{2} \Phi+r \Phi$ reads

$$
\partial_{t} a_{n}=\omega_{n} a_{n}
$$

where the dispersion relation for mode $\omega_{n}$ is given by

$$
\omega_{n}=-D_{0} k_{n}^{2}+r .
$$

The mode amplitudes $a_{n}$ therefore obey $a_{n}=a_{n}(0) \exp \left(\omega_{n} t\right)$ with the prefactors fixed by the initial conditions.

For the empty state to be stable, we require all $\omega_{n}$ to be negative. As the slowest growing mode is $n=0$, this implies $D_{0} k_{0}^{2}<r$, or

$$
L<L_{\mathrm{est}}=\frac{\pi}{2} \sqrt{\frac{D_{0}}{r}},
$$

as discussed in the main text.

\subsection{Steady state at low packing fraction}

To explore the nature of the gaseous phase, it is useful to study the limit of low packing fractions, where the diffusivity takes the form ${ }^{45}$

$$
D(\Phi)=D_{0}(1+\alpha \Phi)+O\left(\Phi^{2}\right) .
$$

The numerical coefficient $\alpha$ measures the leading order change in diffusivity with increasing packing fraction and depends on the shape of the particles as well as their interactions. For repulsive particles, such as our main model system Acetobacter indonesiensis, $\alpha$ is positive. Detailed analytical results are available for hard spheres, yielding $\alpha=1.45^{46}$. Strong attraction can lead to negative $\alpha^{45}$. 
When we include the non-linear packing fraction dependence to leading order, we obtain the following equation of motion

$$
\partial_{t} \Phi(y, t)=D_{0} \partial_{y}^{2}\left(\Phi+\frac{\alpha}{2} \Phi^{2}\right)+r \Phi
$$

If we expand $\Phi(y, t)$ in terms of the normal modes as in Eq. 3, we find

$$
\partial_{t} a_{n}(t)=\omega_{n} a_{n}-\frac{\alpha}{2} D_{0} k_{n}^{2}\left\langle\varphi_{n} \mid \Phi^{2}\right\rangle
$$

where $\left\langle\varphi_{n} \mid \Phi^{2}\right\rangle$ is the projection of $\Phi^{2}(y, t)$ on to the $n$th mode. Close to establishment, we have

$$
\frac{\omega_{0}}{r} \approx 2 \varepsilon \ll 1
$$

in terms of

$$
\varepsilon \equiv \frac{L}{L_{\mathrm{est}}}-1
$$

To leading order in $\varepsilon, \Phi(y, t)=a_{0} \varphi_{0}(y, t)+O\left(\varepsilon^{2}\right)$, which simplifies the amplitude equation Eq. 11 to,

$$
\partial_{t} a_{n}(t)=\omega_{n} a_{n}-\frac{\alpha}{2} D_{0} k_{n}^{2} a_{0}^{2}\left\langle\varphi_{n} \mid \varphi_{0}^{2}\right\rangle
$$

The scalar product on the right hand side evaluates in general to

$$
\begin{aligned}
\left\langle\varphi_{n} \mid \varphi_{0}^{2}\right\rangle & =\frac{2}{L} \int_{0}^{L} d y \cos \left[\left(n+\frac{1}{2}\right) \pi y\right] \cos ^{2}\left(\frac{\pi y}{2}\right) \\
& =\frac{8(-1)^{n}}{\pi\left(3+2 n-12 n^{2}-8 n^{3}\right)} .
\end{aligned}
$$

As before, we confine our attention to the slowest growing mode, $n=0$, for which $\left\langle\varphi_{0} \mid \varphi_{0}^{2}\right\rangle=8 / 3 \pi$ leading to the closed amplitude equation,

$$
\partial_{t} a_{0}=\omega_{0} a_{0}-\frac{4 \alpha}{3 \pi} D_{0} k_{0}^{2} a_{0}^{2}
$$

Rescaling time $\tau=r t$ and using the leading order approximations $D_{0} k_{0}^{2} \approx r+O(\varepsilon)$ and $\omega / r \approx 2 \varepsilon$ (Eq. 12), we obtain

$$
\partial_{\tau} a_{0}=2 \varepsilon a_{0}-\frac{4 \alpha}{3 \pi} a_{0}^{2} .
$$

Up to a shift in time, this logistic differential equation has the simple solution

$$
a_{0}(\tau)=\frac{6 \pi \varepsilon}{4 \alpha} \frac{1}{1+\exp (-2 \varepsilon \tau)}
$$

To interpret this result, let us take hard spheres as an example, for which $\alpha=1.45$ and the packing fraction at the floor of the chamber is about 3.25ع. Extrapolating from this lowest order expansion, we can estimate that a chamber length of no more than $24 \%$ above the establishment length is needed to approach random close packing ( $\Phi_{\text {rcp }} \approx 64 \%$ for monodisperse spheres).

Of particular significance is the relaxation time to the steady state, the inverse of which is taken as a common measure for the resilience of an ecological system ${ }^{47}$. To leading order, this relaxation time is $1 /(2 \varepsilon)$ doublings and thus diverges near the establishment transition. Relaxation thus can take much longer than the diffusive exploration of the chamber, which takes about one cell doubling near the establishment transition $\left(L_{\text {est }}^{2} /\left(2 D_{0} \ln 2\right)=P i^{2} /(8 \ln 2) \approx 1.8\right.$ doublings $)$. Therefore, we generically expect a time scale separation between relaxation of the density profile towards the cosine shape (fast) and relaxation of the amplitude of the cosine (slow).

Although the cells in our experiments are neither spherical nor monodisperse, we expect the above results to apply up to pre-factors. For example, since $\varepsilon$ remains small up to the jamming transition also in our experiments, one would expect long relaxation times throughout the gaseous phase. Indeed, relaxation in our flow shift experiments took up to $10 \mathrm{~h}$ or five doublings (see green curve in Fig. ED5). 


\subsection{Mechanical analogy predicts steady state colonization patterns}

Let us consider the one-dimensional reaction-diffusion equation for the cell packing fraction $\Phi(y, t)$ at position $y$ and time $t$

$$
\begin{aligned}
\partial_{t} \Phi(y, t) & =-\partial_{y} J+b(\Phi) \\
J(y, t) & \equiv-D(\Phi) \partial_{y} \Phi
\end{aligned}
$$

where we allow for an arbitrary density-dependence in both the collective diffusivity $D(\Phi)$ as well as the growth rate $b(\Phi)$. As pointed out in the main text, the linear growth rate in our experimental system is to a good approximation constant, which corresponds to $b(\Phi)=r \Phi$. The mathematical treatment in this section is independent from this simplifying condition.

We define the quantity

$$
\Pi(\Phi) \equiv \int_{0}^{\Phi} d \Phi^{\prime} D\left(\Phi^{\prime}\right)
$$

such that the diffusive current is given by

$$
J=-\partial_{\Phi} \Pi(\Phi) \partial_{y} \Phi=-\partial_{y} \Pi(y)
$$

where we identified $\Pi(y) \equiv \Pi[\Phi(y)]$ to simplify the notation. Equation 23 formally implies that a gradient of $\Pi(y)$ drives a current just like a conventional pressure gradient would. We therefore call $\Pi$ effective pressure. Since for passive diffusion ${ }^{2}$, we must have $\partial_{\Phi} \Pi(\Phi)=D(\Phi)>0$, we can invert the equation of state to obtain a the packing fraction $\Phi(\Pi)$ as function of effective pressure $\Pi$.

Next, combining Eqs. 22, 23 yields at steady state

$$
\partial_{y}^{2} \Pi=-b(\Phi)=-\partial_{\Pi} U(\Pi)
$$

where we defined an effective potential

$$
\begin{gathered}
U(\Pi) \equiv \int_{0}^{\Pi} d \Pi^{\prime} b\left[\Phi\left(\Pi^{\prime}\right)\right] \\
U[\Phi(\Pi)]=\int_{0}^{\Phi(\Pi)} d \Phi^{\prime} D\left(\Phi^{\prime}\right) b\left(\Phi^{\prime}\right) .
\end{gathered}
$$

The boundary conditions imply $\Pi(L)=0$, manifestly ensured through Eq. 22, and $\partial_{y} \Pi(y=0)=0$, which we will account for below.

Notice that Eq. 24 is formally identical to Newton's equation for a particle at position $\Pi(y)$ at time $y$ freely falling in a potential $U(\Pi)$. Hence, we can use the principle of mechanical energy conservation to immediately predict the velocity of the moving particle, which corresponds to the negative current

$$
J(y)=-\partial_{y} \Pi=\sqrt{2\left(U_{0}-U(\Pi)\right)},
$$

manifestly satisfying the no-flux boundary condition $\partial_{y} \Pi(y=0)=0$. Note that we use the notation $U_{0} \equiv U\left(\Pi_{0}\right)$ and $\Pi_{0} \equiv \Pi(y=0)$.

Integrating Eq. 27 yields

$$
\int_{\Pi}^{\Pi_{0}} \frac{d \Pi^{\prime}}{\sqrt{2\left(U_{0}-U\left(\Pi^{\prime}\right)\right)}}=\int_{0}^{y(\Pi)} d y=y(\Pi)
$$

or

$$
\begin{aligned}
y(\Phi) & =\int_{\Phi}^{\Phi_{0}} \frac{d \Phi^{\prime} \partial_{\Phi} \Pi}{\sqrt{2\left(U_{0}-U\left(\Phi^{\prime}\right)\right)}} \\
& =\int_{\Phi}^{\Phi_{0}} \frac{d \Phi^{\prime} D\left(\Phi^{\prime}\right)}{\sqrt{2\left(U_{0}-U\left(\Phi^{\prime}\right)\right)}}
\end{aligned}
$$

where $U(\Phi)=U[\Phi(\Pi)]$, as defined in Eq. 26 .

The integrals in Eq.s 30 and 26 can be solved numerically without problems for any $D(\Phi)>0$ and $b(\Phi)$, provided $U(\Phi)<U_{0}$ along the trajectory.

\footnotetext{
${ }^{2}$ The mechanism of Motility-Induced Phase Separation is based on an active movement (motility), which can generate a negative effective diffusivity ${ }^{34}$.
} 


\subsection{General approach to compute the phase diagram}

Generically, the shape of the potential $U(\Phi)$ will start at $U(\Phi=0)=0$ and increase monotonically because $U^{\prime}=-D(\Phi) b(\Phi) \leq$ 0 , unless we allow for a region of negative net growth $b<0$. The behavior near $\Phi=0$ is quadratic since $U^{\prime}(\Phi=0)=0$ from the no-flux boundary condition and we assume analyticity of $D$ and $b$. For small $\Phi$, we expect $D^{\prime}>0$ suggesting that the potential at first rises faster than a parabola. At larger $\Phi$ we expect, instead, a negative slope of $D(\Phi)$ resulting in a flattening of the potential until jamming leads to rapid rise of the potential. Hence, generically, we may envision the potential energy surface to look as in Fig., which corresponds to the hard sphere case discussed below at greater detail.

To determine the density at the floor of the chamber, we have to solve the following problem: Let a point mass move down this energy landscape from $\Phi=\Phi_{0}$ back down to $\Phi=0$. The time it takes the moving mass to reach $\Phi=0$ has to equal the length $L$ of the chamber. If the sojourn time is too small (large) we have to increase (decrease) $\Phi_{0}$. Mathematically, we can formulate this condition using Eq. 30,

$$
L=F\left(\Phi_{0}\right) \equiv \int_{0}^{\Phi_{0}} \frac{d \tilde{\Phi} D(\tilde{\Phi})}{\sqrt{2\left(U\left(\Phi_{0}\right)-U(\tilde{\Phi})\right)}} .
$$

We have thus obtained an equation that can be used to determine a phase diagram as in Fig. $2 \mathrm{~b}$ from any $D(\Phi)$ and $b(\Phi)$. Multiple steady states exist if there are multiple $\Phi_{0}$ values with identical sojourn times. The trivial case for which this happens is a simple parabola, which corresponds to the case without density-dependence, $D=$ const. and $b(\Phi)=r \Phi$. Then, we have a critical length where any density $\Phi_{0}$ will lead to a marginally stable steady state.

More realistically, multiple steady states occur if the potential fluctuates around a parabola. The density interval supporting multiple steady states is bounded by $\Phi_{0}$-values for which $\partial_{\Phi_{0}} F\left(\Phi_{0}\right)=0$. This condition can be computed explicitely as follows,

$$
\begin{aligned}
0 & =\partial_{\Phi_{0}} F\left(\Phi_{0}\right)=\partial_{\Phi_{0}} \int_{0}^{\Phi_{0}} \frac{d \xi D\left(\Phi_{0}-\xi\right)}{\sqrt{2\left(U\left(\Phi_{0}\right)-U\left(\Phi_{0}-\xi\right)\right)}} \\
& =\frac{D_{0}}{2 U\left(\Phi_{0}\right)}+\int_{0}^{\Phi_{0}} \frac{d \xi D^{\prime}\left(\Phi_{0}-\xi\right)}{\sqrt{2\left(U\left(\Phi_{0}\right)-U\left(\Phi_{0}-\xi\right)\right)}} \\
& +\left(D\left(\Phi_{0}\right) b\left(\Phi_{0}\right)-D\left(\Phi_{0}\right) b\left(\Phi_{0}\right)\right) \int_{0}^{\Phi_{0}} \frac{d \xi D\left(\Phi_{0}-\xi\right)}{\left[2\left(U\left(\Phi_{0}\right)-U\left(\Phi_{0}-\xi\right)\right)\right]^{3 / 2}}
\end{aligned}
$$

where we substituted $\xi \equiv \Phi_{0}-\tilde{\Phi}$ in the first line and using Eq. 26 to express the derivative of $U$ in the last line. If Eq. 33 has any solution there must exist multiple steady states.

\subsection{Collective diffusion for hard spheres}

In general, $D(\Phi)$ is a collective diffusion coefficient as opposed to a tracer diffusivity or self-diffusion coefficient. With purely passive diffusion, $D(\Phi)$ can be decomposed into the product $D(\Phi)=\mu(\Phi) k_{B} T \partial_{\Phi} P(\Phi)$ of two terms with an intuitive interpretation:

- $\mu(\Phi)$ is the collective mobility, a transport coefficient that describes the sedimentation velocity and is typically a dreasing function of packing fraction $\Phi$. As a transport coefficient it depends on the equations of motion, and the treatment of hydrodynamics.

- $P(\Phi)$ is the osmotic pressure of the cell suspension, and its derivative is proportional to the (osmotic) bulk modulus, which has to be positive. Because $P(\Phi)$ is a pure equilibrium quantity, it can be readily obtained from Monte Carlo simulations without modeling the surrounding fluid at all.

For a system of hard spheres, a multipole expansion of the effective hydrodynamic interaction ${ }^{49}$ can be used to extract the collective mobility for particles immersed in an incompressible fluid. With collective mobilities computed using this method, we confirmed the empirical Richardson-Zaki scaling form ${ }^{50}, \mu(\Phi)=\mu_{0}(1-\Phi)^{\eta}$ where $\mu_{0}$ is the particle mobility in the dilute limit. We extracted $\eta=5.8$ from a linear regression of $\mu(\Phi)$. The fit and accompanying data are shown in Fig. 4, which over the range of packing fractions considered are in good agreement. Similarly, the equation of state of hard spheres is known to be well described by a Carnahan-Starling equation. ${ }^{51}$ In terms of the packing fraction, the pressure $P(\Phi)$ is given by, $P(\Phi) / k_{\mathrm{B}} T=(6 \Phi / \pi)\left(1+\Phi+\Phi^{2}-\Phi^{3}\right) /(1-\Phi)^{3}$. Taken together, we obtain the collective diffusion coefficient via $D(\Phi)=k_{\mathrm{B}} T \mu(\Phi) \partial_{\Phi} P(\Phi)$, which was used together with $b(\Phi)=r \Phi$ to determine the hard sphere phase diagram Fig. $2 \mathrm{~b}$ in the main text using Eq. 31. 


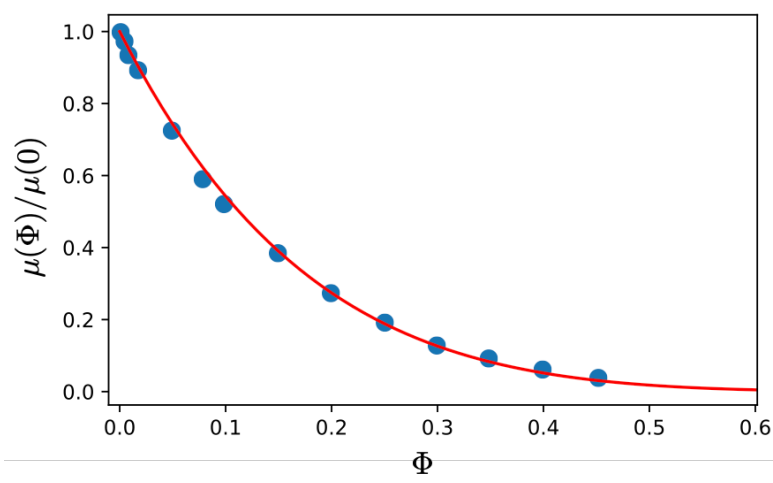

Figure 4. Collective mobility with complete many-body hydrodynamic interactions (blue circles) and a fit to the Richardson-Zaki scaling form (red line).

\section{Proliferating soft disk simulations}

To see which phase transitions emerge in a minimal model that only includes proliferation, cell diffusion and cell repulsion, we explicitly simulate a mechanical system of soft disks that undergo Brownian motion and that divide with a constant rate.

\subsection{Model details}

Each particle, $i$, obeys a stochastic equation of motion for its position in two dimensions, $\mathbf{r}_{i}=\left\{x_{i}, y_{i}\right\}$,

$$
\frac{d \mathbf{r}_{i}}{d t}=-\mu_{0} \nabla_{i} U\left(\mathbf{r}^{N}\right)+\eta_{i}
$$

where $\mu_{0}$ is the time single particle mobility resulting from the surrounding fluid, $\eta_{i}$ is a Gaussian random variable with mean $\left\langle\eta_{i}\right\rangle=0$ and variance $\left\langle\eta_{i}(t) \otimes \eta_{j}\left(t^{\prime}\right)\right\rangle=2 k_{\mathrm{B}} T \mu_{0} 1 \delta_{i j} \delta\left(t-t^{\prime}\right)$ where $k_{\mathrm{B}} T$ is Boltzmann's constant times the temperature of the fluid and is diagonal for each particle and cartesian component. We solve this equation using a standard first order Euler discretization. In addition to Brownian motion, the particles move in response to a potential $U\left(\mathbf{r}^{N}\right)$ that depends on the full configuration of the system, denoted $\mathbf{r}^{N}$. At any time, there are $N$ total particles, with $N_{\mathrm{m}}$ maturated mother particles and $N_{\mathrm{d}}$ growing daughter particles. The interaction potential is decomposable into $U\left(\mathbf{r}^{N}\right)=U_{\mathrm{b}}\left(\mathbf{r}^{N}\right)+U_{\mathrm{r}}\left(\mathbf{r}^{N}\right)+U_{\mathrm{w}}\left(\mathbf{r}^{N}\right)$ where $U_{\mathrm{b}}$ is the bonding potential between mother and daughter particles, $U_{\mathrm{r}}$ is a purely repulsive interparticle interaction and $U_{\mathrm{w}}$ is a confining potential.

The bonding potential is taken to taken to be a simple Hookian spring,

$$
U_{\mathrm{b}}\left(\mathbf{r}^{N}\right)=\sum_{\substack{i \in N_{\mathrm{m}} \\ j \in N_{\mathrm{d}}}}^{N} \frac{\kappa}{2}\left(r_{i j}-b_{i j}\right)^{2} \chi_{i j}
$$

with stiffness $\kappa$, rest length $b_{i j}$, and $\chi_{i j}=1$ if particles $i$ and $j$ are a mother daughter pair and $\chi_{i j}=0$ otherwise. The specific form of the repulsive interparticle potential is taken to be pairwise decomposable

$$
U_{\mathrm{r}}\left(\mathbf{r}^{N}\right)=\frac{1}{2} \sum_{i \neq j=1}^{N} u_{2}\left(r_{i j}\right)\left(1-\chi_{i j}\right)
$$

where the pair potential $u_{2}\left(r_{i j}\right)$, is a WCA potential ${ }^{52}$

$$
u_{2}\left(r_{i j}\right)= \begin{cases}4 \varepsilon\left[\left(\frac{\sigma_{i j}}{r_{i j}}\right)^{12}-\left(\frac{\sigma_{i j}}{r_{i j}}\right)^{6}\right]+\varepsilon & \text { if } r<2^{1 / 6} \sigma_{i j} \\ 0 \quad \text { else } & \end{cases}
$$

where $\varepsilon$ is a characteristic energy scale and

$$
\sigma_{i j}=\frac{1}{2}\left(\sigma_{i}+\sigma_{j}\right)
$$


where $\sigma_{i}$ is the characteristic size of particle $i$. All pairs of particles, excluding bonded mother and daughter pairs, interact with these are excluded volume interactions. Finally, the confining potential restricts the the particles motion to approximately an area $A=L_{x} L_{y}$ by imposing a steep potential at $x=0, x=L_{x}$, and $y=0$. Specifically, the external wall potential has the form,

$$
U_{\mathrm{w}}\left(\mathbf{r}^{N}\right)=\sum_{i=1}^{N} a\left[e^{-y_{i} / \xi}+e^{-x_{i} / \xi}+e^{-\left(L_{x}-x_{i}\right) / \xi}\right]
$$

with $a$ the amplitude of the confining potential and $\xi$ its characteristic length scale.

The mechanical system outlined above is conservative and describes the motion of a collection of overdamped particles with a simplified, local description of hydrodynamics. ${ }^{53}$ At long times, absent added external forces, its evolution would be consistent with thermal distribution and would conserve particle number. In order to model the growth of the bacterial population and division of an individual mother daughter pair, we endow the daughter particles with a time dependent effective size through the deterministic equation of motion

$$
\frac{d \sigma_{i}(t)}{d t}= \begin{cases}k & \text { for } t<1 / k \\ 0 & \text { else }\end{cases}
$$

with boundary condition $\sigma_{i}(0)=0$ and growth rate $k$. Similarly, to model the budding of the daughter from the mother, the rest distance $b_{i j}$ changes in time with the deterministic equation of motion

$$
\frac{d b_{i j}(t)}{d t}= \begin{cases}k & \text { for } r_{i j}<\sigma_{i} \\ 0 & \text { else }\end{cases}
$$

with boundary condition $b_{i j}(0)=0$ and the same growth rate $k$. When then impose that when the distance between the mother-daughter pair $r_{i j}(t)$ has grown past the effective size of the mother particle, $\sigma_{i}$, we sever the bond potential by $\chi_{i j} \rightarrow 0$ between mother-daughter pair $i j$, provided $t>1 / k$. An illustration of this criteria is shown in Fig. 5. This growth rule results in an increasing excluded area of the mother-daughter pair that grows approximately linearly in time. All of the results in the main text and below employ this rule with a fixed $k$. Growth rules that affect in an exponential increase in the excluded area with time yield qualitatively similar results. Further the results presented are for a fixed growth rate $k$, but generalizations for populations evolving with a distribution of growth rates are also qualitatively similar, provided the distribution is narrow. Synchronized with the bond breaking event, we add new daughter sites to each of the newly divided particles and reinitialize the size and rest length to 0 for each of them. This last step breaks particle number conservation. An illustration of the subsequent increase in $N_{\mathrm{m}}$ is also shown in Fig. 5, which absent mitigating factors will grow exponentially in time.

Finally, consistent with the experiments, we apply an absorbing boundary condition at $y=L_{y}$. The absorbing boundary condition and particle number growth balance at steady-state, resulting in a mean particle number that depends on the geometry and model parameters. We adopt a unit system where $\mu=\varepsilon=k_{\mathrm{B}} T=1$, and mature particles sizes $\sigma=1$. This implies that lengths are defined in multiples of the mature particles size, $x \rightarrow x / \sigma$ and times in units of the diffusion time for an isolated particle to move its diameter, with $D_{0}=k_{\mathrm{B}} T \mu_{0}$ and accompanying units of time, $t \rightarrow t /\left(D_{0} \sigma^{2}\right)$. The stiffness $\kappa$ in principle allows for mechanocoupling between the division and local packing environment, with deterministic division occurring when $\kappa$ is much larger then the local stress on the mother-daughter pair. In this work, we consider this limit and take $\kappa=100$. The confining potential parameters are taken as $a=10$ ans

$$
\xi=1
$$

\subsection{Nonequilibrium phase diagram}

We have studied the particle based model described above and have found qualitative agreement with both the experimental results and simplified theory presented in the main text. Specifically, we have studied a system with confinement defined by fixed spatial scale $L_{x}=15$ and $L_{y}=40$, seeded with an $N_{\mathrm{m}}=10$ initial mother particles. We have found that 10 division cycles is sufficient to relax to a steady state density in the chamber, and evaluated expectation values by averaging over a minimum of 30 additional division cycles. Further, 3-5 independent simulations are used for each expectation value reported. Studies of the effective reaction diffusion model suggests that the function dependence of the system on the length of the chamber enters relative to the establishment length $L_{\text {est }}=\pi \sqrt{D_{0} / 4 k}$, so rather then studying different $L_{y}$ 's, we fixed $L_{y}=L$ and study the dependence on the division rate $k$, and thus $L_{\text {est }}$.

First, we studied the stead-state density distribution in the chamber. For $L / L_{\text {est }}<1$, as expected the density in the chamber is 0 at steady-state on average. For $L / L_{\text {est }}>1$, the particles are able to colonize the chamber, and evolve a stationary 

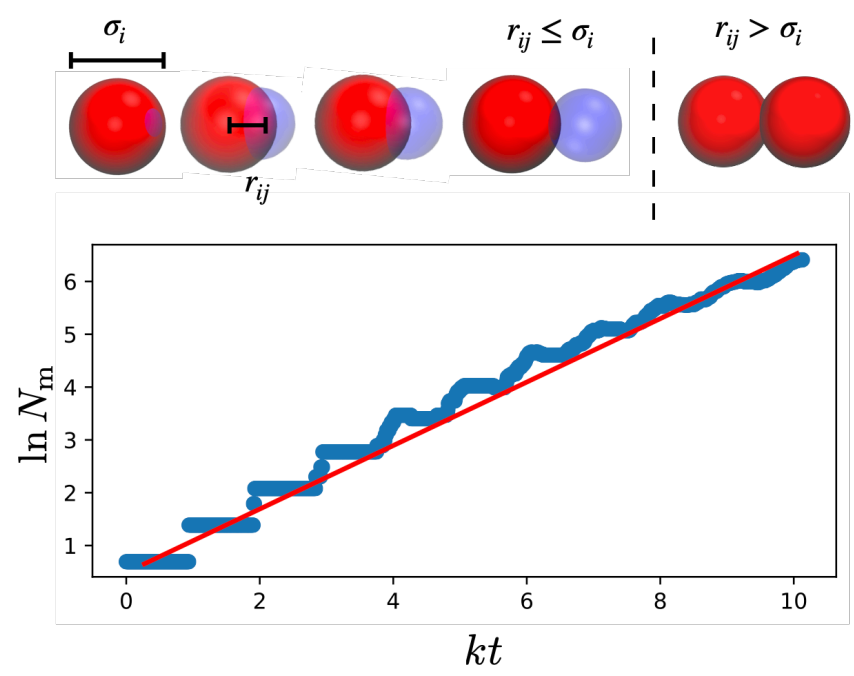

Figure 5. Illustration of the growth and division. (top) Illustration of the mechanical model of division of a mother (red) daughter (blue) particle pair, where the charactoristic size of the mother is $\sigma_{i}$ and its displacement from a daughter is $r_{i j}$ (bottom) Illustration of the subsequent exponential proliferation of particles in time over 10 division times. The red line is a guide to the eye.

density distribution. The distribution can be characterized analogously through local packing fraction $\Phi(y)=\rho(y) \pi / 4$ where $\hat{\rho}(y)=\left\langle\delta\left(y-y_{i}\right)\right\rangle$. Generally, the discrete size of the particles relative to a flat wall will result in an oscillatory density profile when the overall density is larger, $\rho>0.1$, which is a result of density correlations induced by their excluded area. Such an oscillatory density density profile is not predicted by the simple reaction diffusion model employed in the main text. In order to make contact with that perspective, we report in Fig 6 density profiles coarse-grained over a the length-scale of the particle ${ }^{54}$. We achieve this using by convoluting the number density with a Gaussian,

$$
\rho(y)=\int d y^{\prime} \hat{\rho}\left(y^{\prime}\right) \frac{e^{-\left(y-y^{\prime}\right)^{2} / 2 \sigma^{2}}}{\sqrt{2 \pi \sigma^{2}}}
$$

which smooths the profile out. Further, we consider the contribution of the density only from the mother particles, and evaluate expectation values for times that are integer multiples of $1 / k$.

Figure 6 specifically reports conditions for $L / L_{\text {est }}=\{1.2,2,4\}$. We also compare those calculations to the predictions of the reaction diffusion model. For $L_{y} / L_{\text {est }}=1.2$, the density is small enough that we find good agreement with the predicted cosine profile, $\Phi(y)=\Phi(0) \cos \left(\pi y / 2 L_{y}\right)$. At elevated $L / L_{\text {est }}$, deviations of the cosine profile are found and specifically at $L_{y} / L_{\text {est }}=4$, the distribution is flat in the interior of the chamber with an exponential boundary layer that brings the packing fraction to 0 at $y=L$. Using a parameterization of the collective diffusion constant $D(\Phi)$, evaluated by computing the packing fraction dependent mobility $\mu(\phi)$ that is well described by $\mu(\phi) \approx \mu_{0} \exp (-1.70 \Phi-0.18 \Phi /(1-1.33 \Phi))$, and the equation of state well described by $P(\Phi) / k_{\mathrm{B}} T \approx 1.27 \Phi+2.55 \Phi^{2}-9.35 \Phi^{3}+42.72 \Phi^{4}$ of the WCA disks, consistent with previous estimations, ${ }^{55,56}$ we can numerically solve the reaction diffusion equation and determine the packing fraction profile for $L / L_{\text {est }}=4$. This predicted profile is in good agreement with the coarse-grained profile from the simulations.

To estimate the boundaries between the extinct, established, and jammed phases, we have computed the coarse-grained value of the packing fraction at the wall as a function of $L / L_{\mathrm{est}}$. This is shown in Fig. 7. As anticipated by the reaction diffusion analysis, the establishment transition occurs for $L / L_{\mathrm{est}} \approx 1$, which is consistent with our findings that $L<L_{\mathrm{est}}$ the chamber is empty at steady-state. For $L>L_{\text {est }}$ the maximum packing fraction gradually increases until $L=L_{\mathrm{jam}} \approx 3.3$ where for this two-dimensional system we find an abrupt change in the maximum packing fraction. The amplitude of this change is small, reflecting the small change in the density upon freezing of the WCA disks, which is around $2 \%{ }^{57}$ Indeed, the pressure measured at the wall at $y=0$, computed from the average force per unit length of wall, $p_{\mathrm{w}}=-\sum_{i}^{N}\left\langle d U_{\mathrm{w}} / d y_{i}\right\rangle / L_{x}$, surpasses the coexistence pressure for the freezing of WCA spheres at this value of $L$. As illustrated in Fig. 2 in the main text, for $L>L_{\text {jam }}$ the system exhibits noticeable crystallinity.

\subsection{Self diffusion calculations}

In order to estimate the self-diffusion coefficient in the chamber as a function of $L / L_{\text {est }}$, we consider only the diffusivity in the $x$ direction, the direction orthogonal to the open end. This is because there exists a net mass current in the $y$ direction, so motion 


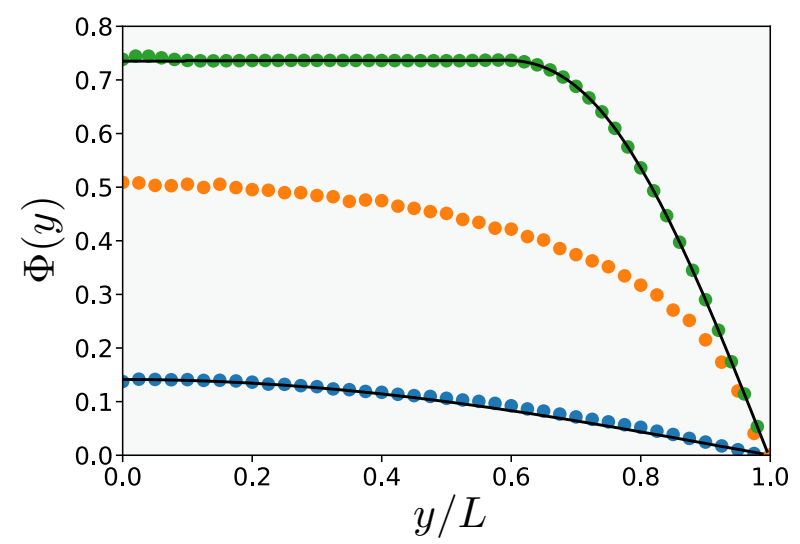

Figure 6. Coarse-grained packing fraction profiles computed from simulations at $L / L_{\mathrm{est}}=\{1.2,2,4\}$ (blue, orange and green) compared to the analytical predictions of the reaction diffusion model (solid lines).

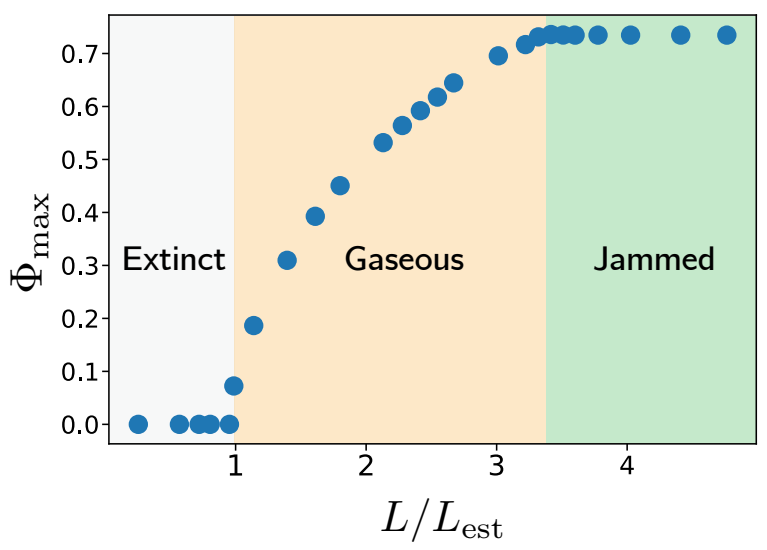

Figure 7. Phase diagram for the proliferating soft disks determined by the maximum coarse-grained packing fraction in the chamber.

is convective in that direction rather than diffusive. For diffusion in the $x$, we use the standard definition

$$
D_{s}(\Phi)=\lim _{t \rightarrow \infty} \frac{1}{2 t}\left\langle\left[x_{i}(t)-x_{i}(0)\right]^{2}\right\rangle
$$

as the long time limit of the mean squared displacement. However two difficulties arise in applying this relation to extract the self diffusion. First, particles leave the chamber at a finite rate, due to the absorbing boundary condition at $y=L$. This requires that we average particles' displacements only up to their lifetime in the chamber. In order to gather sufficient statistics and increase the lifetime of individual particles, we use a larger chamber $L_{y}=L=80$. Second, the finite size of the chamber in the $x$ direction, $L_{x}$ means that particles will only exhibit diffusive dynamics for mean squared displacements $\sqrt{\left\langle\left[x_{i}(t)-x_{i}(0)\right]^{2}\right\rangle}<L_{x} / 2$, and thus the long time limit strictly goes to 0 . We find using $L_{x}=30$ to be large enough that there exists a sufficient separation of timescales between the onset of diffusive motion and its reduction due to confinement that we easily extract a pseudo-time independent self-diffusion constant. This data is reported in Fig. 2.

\section{Theory of Invasion}

For a foreign strain to invade a pre-occupied chamber, an invader has to overcome two hurdles: \# 1) Infiltration: the invader diffuses from the outside against the gradient to reach a favorable position near the floor of the chamber. \# 2) Take over: 
the invader's descendants displace the resident population through a combination of chance (genetic drift) and competitive advantage.

Our experimental observations in combination with our simulations indicate that the main reason for the colonization resistance is the difficulty for outside cells to diffusively penetrate chambers that are already filled. Infiltration is rare even in gaseous chambers, but nearly impossible in jammed populations where the a narrow strip of founder cells at the floor of the chamber is insulated from the outside by diffusion barrier of descendant cells. Accordingly, the biggest impact of the antibiotic occurs in cavities that, due to the growth rate detriment of the resident, become unjammed and hence invadable (Fig. 3a). The larger the growth rate detriment, the larger the range of chamber length that are driven across the tipping point.

In the limit of weak selection, and large chamber population size, infiltration happens on a much faster time scale than take over. This allows to analyze the dynamics in two separate steps. The second, takeover, step is familiar from well-mixed populations. The rate of successful take over depends on the competition between selective advantage of the faster growing invader and genetic drift. The first challenge, however, uniquely depends on the spatial structure of the colonized cavities, which is why we mainly focus on the infiltration step.

The infiltration step is best analyzed backward in time. As we follow the lineage of a randomly chosen cell backward in time, it is advected towards the floor of the chamber, reflecting the intuitive location advantage discussed above. A balance between self-diffusion and advection leads to a steady state lineage distribution, whose extent scales as the ratio of self-diffusivity and advection velocity. While the advection velocity is very similar between gaseous and jammed populations, self-diffusion differs dramatically, by four orders of magnitude, compressing the ancestor distribution in the jammed population to just few cell layers at floor of the chamber.

Our mathematical analysis in the next section shows that the neutral invasion success of an injected invader is proportional to $1 / N$ times the ratio of the invader density in the supply channel and the mean density of the population. This shows that gaseous population do enjoy some colonization resistance, compared to well-mixed populations for which the neutral invasion success would just be $1 / N$.

Yet, the colonization resistance of gaseous populations still is weak compared to partially-jammed populations. Their infiltration is nearly impossible, as it is exponential in the ratio of the thickness of the jammed fraction and the thickness of the founder population, which is at most several cell diameter. The founder population at the floor of the chamber is essentially isolated from the supply channel, through the constant shedding of jammed cells acting as diffusion barrier for any invader. Invasion can only be achieved if this diffusion barrier is broken down, say by an antibiotic treatment or chamber deformation or increase in chamber flow.

Finally, we discuss the case where the growth rate of the resident is reduced by the action of an antibiotic, as done in our experiments. Invadable, gaseous, populations become invaded at rate that is increased by factor of $N_{e} s$, where $s \ll 1$ is the growth rate defect and $N_{e} \gg 1$ is the effective population size of the chamber. The situation is similar to well-mixed populations. The only difference is that, due to the spatial structure of the population, $N_{e} / N<1$ is mildly and strongly reduced in the gaseous and jammed states, respectively.

However, the biggest impact of the antibiotic occurs in cavities that, due to the growth rate detriment of the resident, become unjammed and hence invadable, see Fig. 3. The larger the growth rate detriment, the larger the range of chamber length that are driven across the tipping point.

\subsection{Tracking lineages backward in time}

To model infiltration and neutral take over, we generalize an analysis of "gene surfing" 58 to include a distinction between collective diffusion and self-diffusion. Suppose we sample a cell at position $\xi$ at present time $\tau$ and seek to determine the probability density $G(y, t \mid \xi, \tau)$ that the ancestor of the cell was at position $y$ at earlier time $t$. $G$ then describes backward in time the position of the cell's lineage, which is subject to self-diffusion and advection (no proliferation). $G$ therefore satisfies a generalized diffusion or Fokker-Planck equation, which takes the form

$$
\begin{aligned}
\partial_{t} G(y, t \mid \xi, \tau) & =-\partial_{y} j_{l}(y, t) \\
j_{l}(y, t) & =-D_{s} \partial_{y} G-\left(v_{g}+v_{s}\right) G \\
v_{g} & \equiv j_{p} / c \\
v_{s} & \equiv-D_{s} \partial_{y} \ln (c),
\end{aligned}
$$

where $D_{s}$ is the self-diffusivity and the particle current $j_{p}$ is given by

$$
j_{p}=-D_{g} \partial_{y} \ln (c)+v_{p}
$$

where $v_{p}(y, t)$ is the particle velocity at $(y, t)$ due to any external force. 
The key part here is the contribution of the lineage current due to self-diffusion. The mathematical form $v_{s}=-D_{s} \partial_{y} \ln (c)$ is fixed by the requirement that, for $v_{g}=0, G \propto c$ must be a steady sate solution with vanishing current.

Suppose, the chamber population has reached a steady state and is large enough so that we can ignore density fluctuations. Assuming there is no external force, $v_{p}=0$, the steady state of the ancestor distribution is then given by

$$
G(y)=c(y)^{1+\frac{D_{g}}{D_{s}}} / \mathscr{N},
$$

where $\mathscr{N}$ is a normalizing factor,

$$
\mathscr{N}=\int_{y=0}^{L} d y c(y)^{1+\frac{D_{g}}{D_{s}}}
$$

From this, we can conclude that one particle injected at $y$, will after relaxation in the cavity take up a fraction

$$
u(y)=c(y)^{\frac{D_{g}}{D_{s}}} / \mathscr{N} .
$$

This expression is particularly useful for the gaseous phase where gradient- and self-diffusivity are nearly identical. In this case, we have an approximately cosine density profile and, with $D_{g}=D_{s}$, we obtain

$$
u(y) \approx \frac{8}{\pi^{2}} \frac{c(y)}{\bar{c}} \frac{1}{N},
$$

where $\bar{c}=N / L$ is the mean population density in the chamber. This expression shows that the neutral invasion success is much less than the well-mixed expectation $1 / N$ because the invading cell has to enter from the supply where the cell density is very low.

In the case of a jammed population, the above expressions are not so useful because they require us to know the minute spatial density variations in the jammed phase. Instead, we can exploit the fact that the density hardly varies apart from a boundary layer near the exit of the chamber. A vanishing particle current at steady state requires

$$
-D_{g} \partial_{y} c(y, t)=r \int_{0}^{y} d y^{\prime} c\left(y^{\prime}, t\right) \approx r c_{0} y,
$$

where we used $c(y, t) \approx c_{0}$ in the last step. Hence, the steady state ancestor distribution is a decaying Gaussian,

$$
G(y)=\exp \left[-\frac{r}{2 D_{s}}\left(1+\frac{D_{s}}{D_{g}}\right) y^{2}\right] / \mathscr{N} .
$$

Since $D_{s} / D_{g} \ll 1$ in the jammed phase, we conclude that fixation becomes small very rapidly when $y>\lambda_{s} \equiv \sqrt{2 D_{s} / r}$.

In our experimental system, we found that $\lambda_{s}$ is about one cell diameter and the thickness of the jammed fraction was about 50 cell diameters, even just after the tipping point. It is, therefore, appropriate to think of the jammed populations as diffusively isolated from the outside environment.

\section{SI References}

38. Ryuichi Yamada, Sonali A Deshpande, Kimberley D Bruce, Elizabeth M Mak, and W Ja William. Microbes promote amino acid harvest to rescue undernutrition in drosophila. Cell reports, 10(6):865-872, 2015.

39. Benjamin Obadia, Zehra Tüzün Güvener, Vivian Zhang, Javier A Ceja-Navarro, Eoin L Brodie, W Ja William, and William B Ludington. Probabilistic invasion underlies natural gut microbiome stability. Current Biology, 27(13):19992006, 2017.

40. Christopher J Marx and Mary E Lidstrom. Development of improved versatile broad-host-range vectors for use in methylotrophs and other gram-negative bacteria. Microbiology, 147(8):2065-2075, 2001.

41. J Cooper McDonald, David C Duffy, Janelle R Anderson, Daniel T Chiu, Hongkai Wu, Olivier JA Schueller, and George M Whitesides. Fabrication of microfluidic systems in poly (dimethylsiloxane). ELECTROPHORESIS: An International Journal, 21(1):27-40, 2000.

42. William Thielicke and Eize J Stamhuis. Pivlab-time-resolved digital particle image velocimetry tool for matlab. Published under the BSD license, programmed with MATLAB, 7(0.246):R14, 2014.

43. Jean-Yves Tinevez, Nick Perry, Johannes Schindelin, Genevieve M Hoopes, Gregory D Reynolds, Emmanuel Laplantine, Sebastian Y Bednarek, Spencer L Shorte, and Kevin W Eliceiri. Trackmate: An open and extensible platform for single-particle tracking. Methods, 115:80-90, 2017. 
44. Chujiro Matano. On the relation between the diffusion-coefficients and concentrations of solid metals. Japanese Journal of Physics, 8:109-113, 1933.

45. William Bailey Russel, WB Russel, Dudley A Saville, and William Raymond Schowalter. Colloidal dispersions. Cambridge university press, 1991.

46. GK406082 Batchelor. Brownian diffusion of particles with hydrodynamic interaction. Journal of Fluid Mechanics, 74(1):1-29, 1976.

47. Crawford S Holling. Resilience and stability of ecological systems. Annual review of ecology and systematics, 4(1):1-23, 1973.

48. Michael E Cates and Julien Tailleur. Motility-induced phase separation. Annu. Rev. Condens. Matter Phys., 6(1):219-244, 2015.

49. Anthony JC Ladd. Hydrodynamic transport coefficients of random dispersions of hard spheres. The Journal of chemical physics, 93(5):3484-3494, 1990.

50. JF Richardson and WN Zaki. Sedimentation and fluidisation: Part i: Transactions of the institution of chemical engineers, v. 32, p. S82-S100, 1954.

51. Norman F Carnahan and Kenneth E Starling. Equation of state for nonattracting rigid spheres. The Journal of chemical physics, 51(2):635-636, 1969.

52. John D Weeks, David Chandler, and Hans $C$ Andersen. Role of repulsive forces in determining the equilibrium structure of simple liquids. The Journal of chemical physics, 54(12):5237-5247, 1971.

53. Robert Zwanzig. Nonequilibrium statistical mechanics. Oxford University Press, 2001.

54. Adam P Willard and David Chandler. Instantaneous liquid interfaces. The Journal of Physical Chemistry B, 114(5):1954$1958,2010$.

55. A Mulero, F Cuadros, and C Galán. Test of equations of state for the weeks-chandler-andersen reference system of two-dimensional lennard-jones fluids. The Journal of chemical physics, 107(17):6887-6893, 1997.

56. Alice L Thorneywork, Roberto E Rozas, Roel PA Dullens, and Jürgen Horbach. Effect of hydrodynamic interactions on self-diffusion of quasi-two-dimensional colloidal hard spheres. Physical review letters, 115(26):268301, 2015.

57. Shubhendu Shekhar Khali, Dipanjan Chakraborty, and Debasish Chaudhuri. Two-step melting of the weeks-chandleranderson system in two dimensions. Soft Matter, 2021.

58. Oskar Hallatschek and David R Nelson. Gene surfing in expanding populations. Theoretical population biology, 73(1):158170, 2008.

59. Marie-Cecilia Duvernoy. Growth mechanics of a bacterial microcolony. $\mathrm{PhD}$ thesis, Université Grenoble Alpes, 2015.

60. Claudio Aguilar, Hera Vlamakis, Alejandra Guzman, Richard Losick, and Roberto Kolter. Kind is a checkpoint protein linking spore formation to extracellular-matrix production in bacillus subtilis biofilms. MBio, 1(1), 2010.

61. Jing Yan, Carey D Nadell, Howard A Stone, Ned S Wingreen, and Bonnie L Bassler. Extracellular-matrix-mediated osmotic pressure drives vibrio cholerae biofilm expansion and cheater exclusion. Nature communications, 8(1):1-11, 2017.

62. Oscar P Kuipers, Pascalle GGA de Ruyter, Michiel Kleerebezem, and Willem M de Vos. Quorum sensing-controlled gene expression in lactic acid bacteria. Journal of Biotechnology, 64(1):15-21, 1998. 


\section{Extended data}

\begin{tabular}{|c|c|c|c|c|c|}
\hline Species & Strain & Note & Medium & Scale-dependent colonization & Reference \\
\hline \hline Escherichia coil & MG1655 & & LB & Not observed & \\
Escherichia coil & UG2441 & Less adhesive & LB & Not observed & 59 \\
Bacillus subtilis & HV1235 & Matrix mutant & LB & Not observed & 60 \\
Vibrio cholerae & JY287 & Matrix mutant & LB & Observed & 61 \\
Acetobacter pasteurianus & LFM13 & Fly-gut derived & MRS & Observed & 28 \\
Acetobacter tropicalis & LFM14 & Fly-gut derived & MRS & Observed & 28 \\
Lactococcus lactis & NZ9000 & & GM17 & Observed & 62 \\
\hline
\end{tabular}

Table 1. Strains cultured in our panflute devices. Scale-depedent colonization was observed across multiple species (see Fig. ED8), but was not observed for strains which were highly adhesive to walls or had a strong capability of bioflim formation or filamentation. 
a)

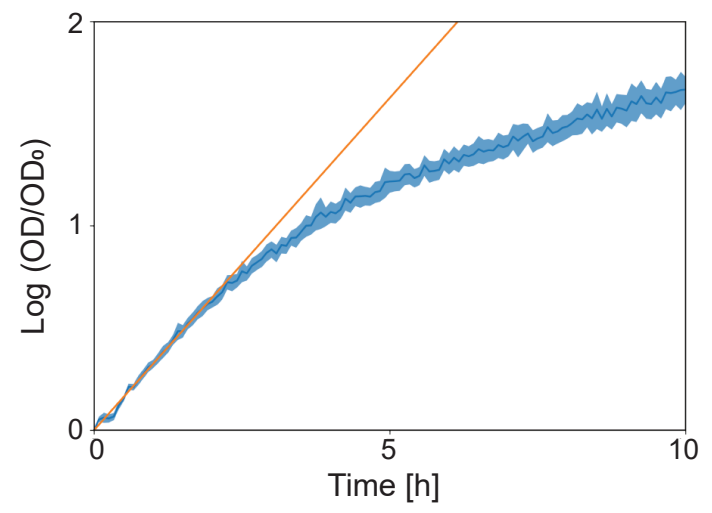

c)

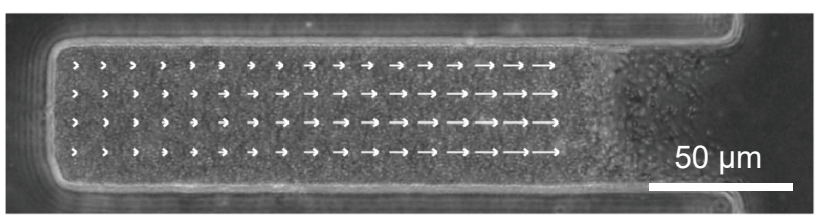

b)

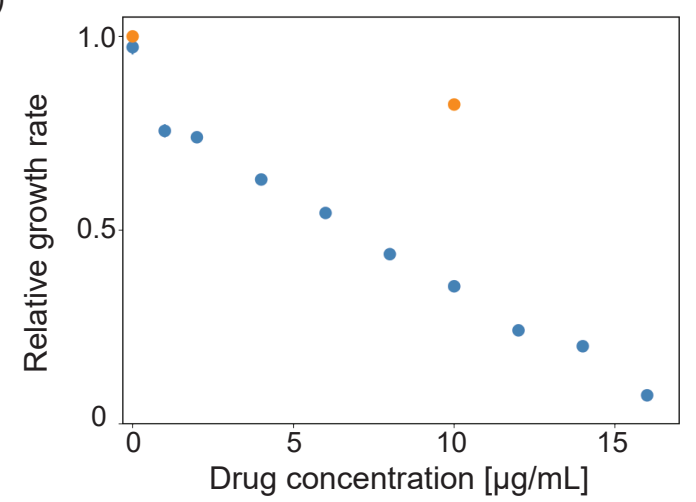

d)

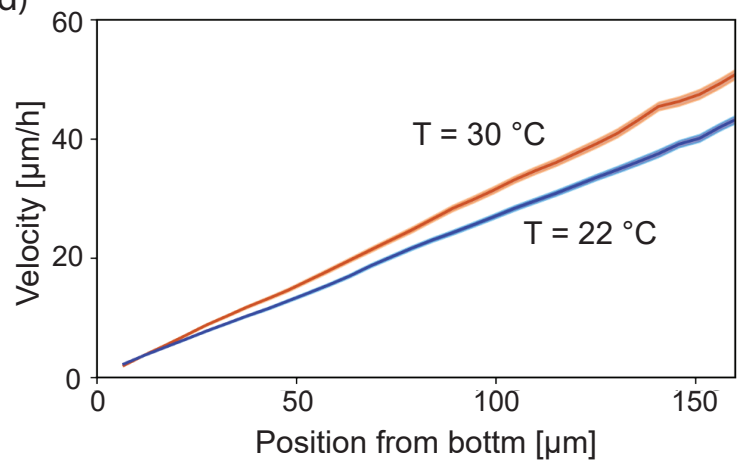

Figure ED1. Growth rate measurements with a plate reader and particle image velocimetry (PIV). (a) The growth rate of Acetobacter indonesiensis is measured with a plate reader. The maximum growth rate at $30{ }^{\circ} \mathrm{C}$ is estimated as $0.325 \pm 0.003$ [1/h] from the initial growth of 4 independent populations. (b) The growth rate of tetracycline-sensitive (blue) and resistant (orange) cells are measured with various drug concentrations and normalized by the the growth rate of drug-resistant cells in the absence of the drug. The minimum inhibitory concentration is estimated about $17 \mu \mathrm{g} / \mathrm{mL}$ by extrapolating the plot. The averaged growth rate for each condition is calculated from 4 replicates. (c) A schematic of PIV analysis. Arrows show the local velocity of the positions. The length of arrows is proportional to the local velocity. (d) The local velocities of cells at high temperature (red, $30^{\circ} \mathrm{C}$ ) and low temperature (blue, $22{ }^{\circ} \mathrm{C}$ ) are linear functions of the position from the bottom of a microfluidic chamber. The solid lines are the averaged local velocities over 3 hours, and the shaded regions show the standard error of mean. The growth rate of cells can be derived from the slope of the linear function as $0.332 \pm 0.007$ and $0.280 \pm 0.001$ $[1 / \mathrm{h}]$ at high and low temperature respectively. 
a)

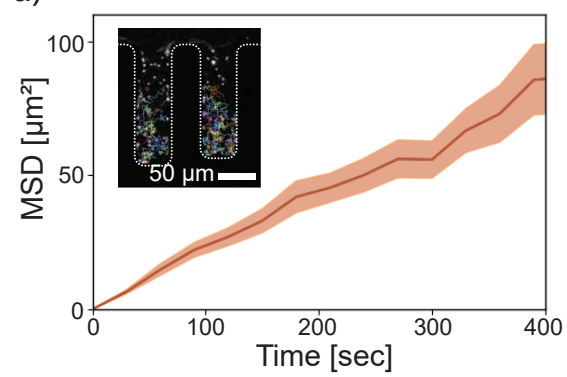

b)

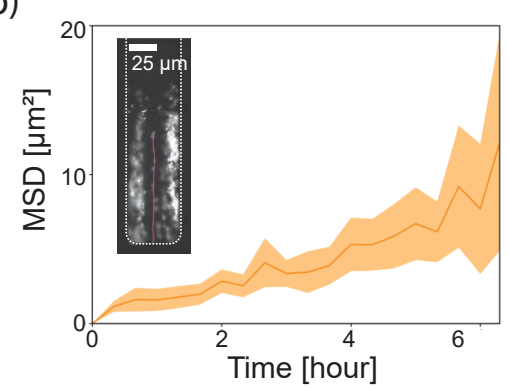

c)

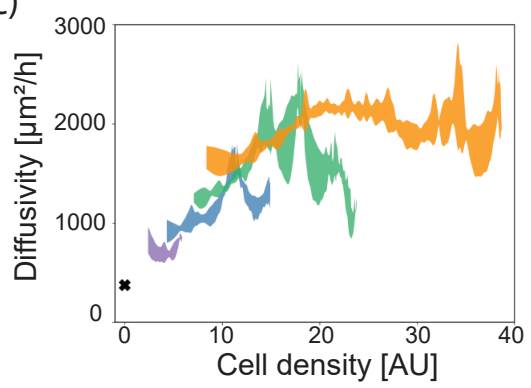

Figure ED2. Measuring self-diffusivity and collective diffusivity. (a) Self diffusivity is measured by tracking single cells in gaseous phases (inset: a snapshot of single-cell tracking). The self diffusivity is calculated as $376 \pm 6 \mu \mathrm{m}^{2} / \mathrm{h}$ from the mean square displacements in the horizontal direction. The error is estimated from fitting. (b) Diffusivity in jammed phases is estimated by manually tracking lineages. The diffusivity is calculated as $0.62 \pm 0.02 \mu \mathrm{m}^{2} / \mathrm{h}$ from the mean square displacements in the horizontal direction. (c) Collective diffusivity is calculated from steady state density profiles (Method 1.9.2) of gas phases in 4 chambers with various depth (the colors show different chambers in the same panflute). The measured collective diffusivity shows a trend of unimodality. The black cross shows the self diffusivity measured in (a). The errors are estimated from the smoothing parameters.

a)

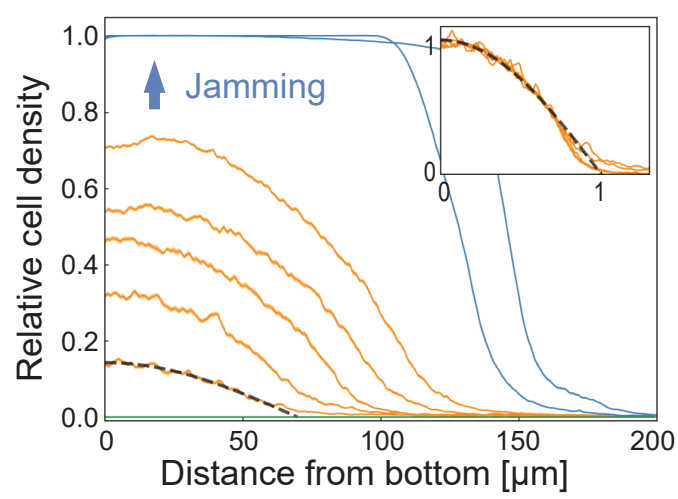

b)

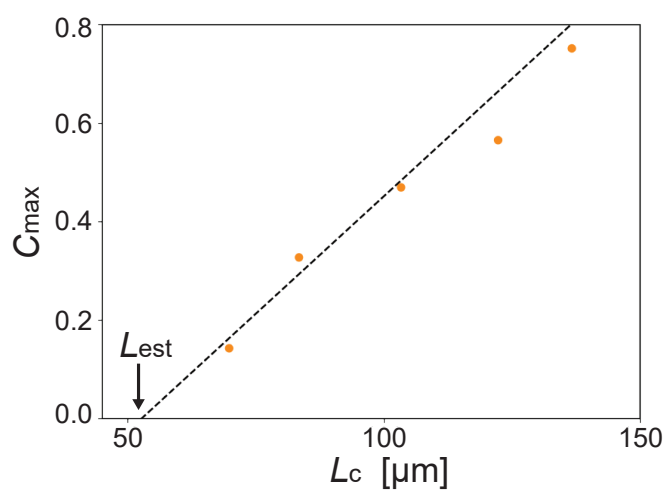

Figure ED3. Density profiles of gaseous phases can be scaled to approximately collapse onto a master curve. (a) The steady-state density profiles in a Microfluidic Panflute device. The plot is taken from Fig. 1d. The density profiles in the gaseous state can be well approximated by the function $c_{\max } \cos \left(\pi x / 2 L_{c}\right)$, which can be seen in the rescaled plot showing $c / c_{\max }$ vs. $x / L_{c}$ (inset). (b) Plotting $L_{c}$ vs. $c_{\max }$ yields a near linear relationship in the gaseous state. Extrapolating the linear fitting of the lowest three points to vanishing density yields an estimate of the establishment length $L_{\text {est }} \approx 53 \pm 7 \mu \mathrm{m}$. The error is estimated from fitting. By comparison, our linear stability analysis predicted $L_{\mathrm{est}} \approx 53 \pm 1 \mu \mathrm{m}$ (see main text). 
a)

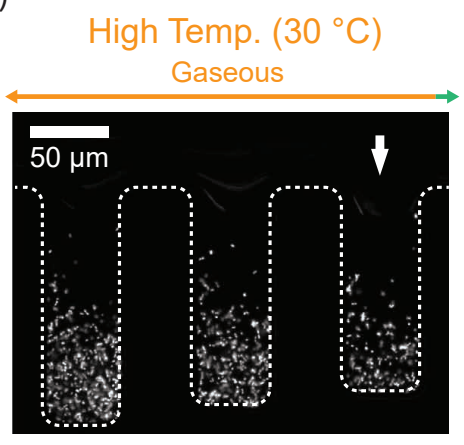

Low temp. $\left(22^{\circ} \mathrm{C}\right)$ Gaseous Empty

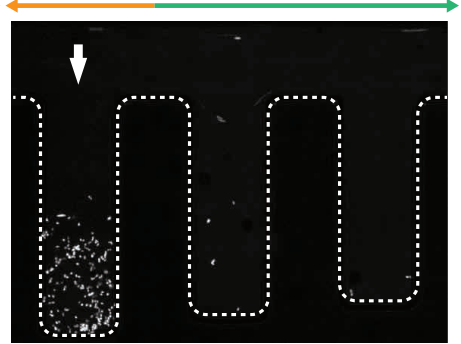

b)

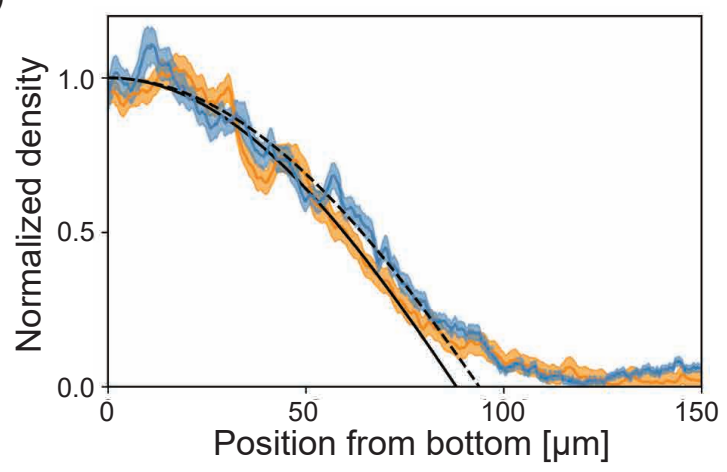

Figure ED4. The establishment length $\ell_{\text {est }}$ shifts upon temperature change. (a) The onset of population establishment at high $\left(30{ }^{\circ} \mathrm{C}\right)$ and low $\left(22{ }^{\circ} \mathrm{C}\right)$ temperature. The incubation temperature was changed from $30{ }^{\circ} \mathrm{C}$ to $22{ }^{\circ} \mathrm{C}$ after the system reached a steady state (see Methods). The critical establishment lengths (white arrows) were measured. (b) The shift of the critical establishment length. The steady state density profile at high (orange) and low (blue) temperature was fitted by a cosine function (black solid and dashed lines, respectively) and normalized. The establishment length was defined by the x-intercept. The relative change of this critical length $(6.6 \%)$ was consistent with our theory predicting it to be given by the square root of the relative growth rate change $(8.6 \%$, Fig. ED1d).

a)

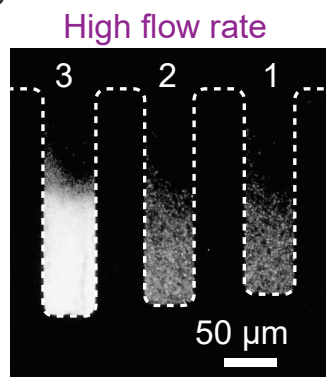

$t=15 \mathrm{~h}$
Low flow rate

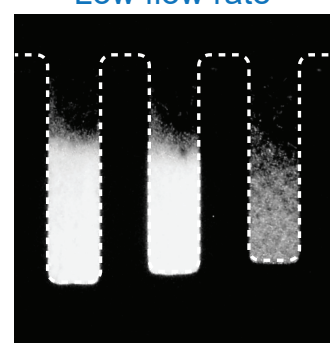

$t=60 \mathrm{~h}$
High flow rate



$t=90 \mathrm{~h}$ b)

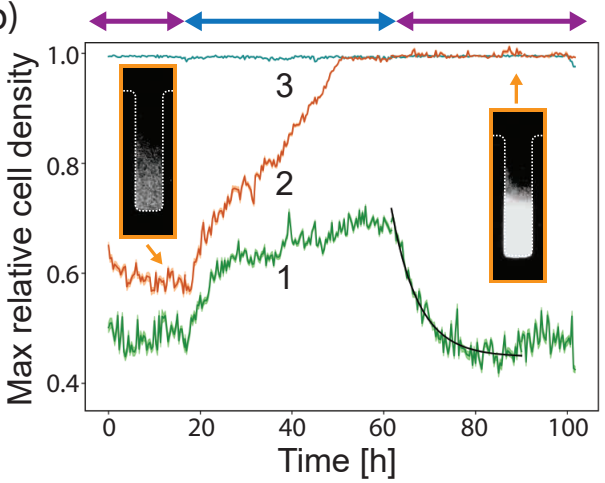

Figure ED5. Bistability in an intermediate-sized pipe. (a) Populations in a Microfluidic Panflute were perturbed by decreasing flow rate for about 40 hours, that extended effective chamber depths. The chamber 2 exhibited a bistability upon the perturbation while the two adjacent chambers ( 1 and 3 ) showed only one stable state. (b) Dynamics of the max cell density upon the flow rate perturbation. Two representative shapshots for each stable state of the chamber 2 are shown in the insets. The black line shows an exponential fit to the population decay. The decay time is $5.9 \pm 0.4$ hour (the error is estimated from fitting). 
bioRxiv preprint doi: https://doi.org/10.1101/2021.05.13.444017; this version posted May 15, 2021. The copyright holder for this preprint (which was not certified by peer review) is the author/funder, who has granted bioRxiv a license to display the preprint in perpetuity. It is made available under aCC-BY-NC 4.0 International license.

a)

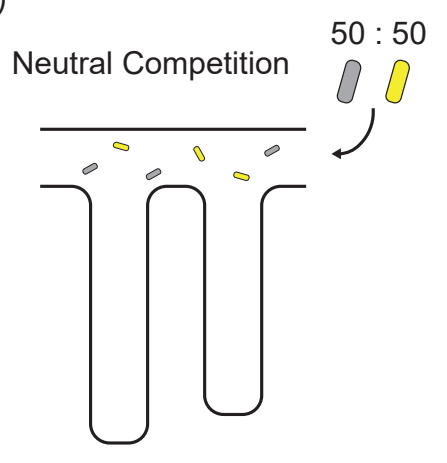

b)

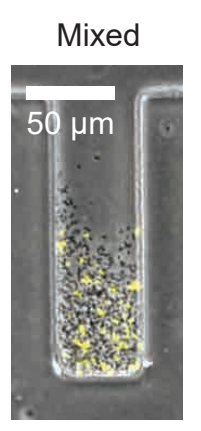

c)

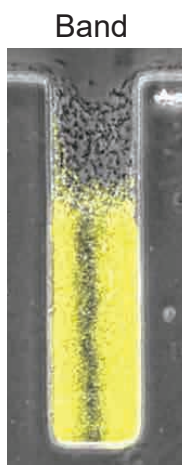

d)

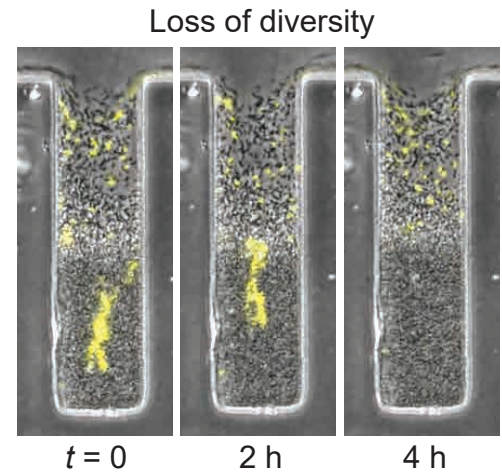

Figure ED6. Neutral dynamics of mixed cultures reveal suppressed lineage diffusion in jammed population. (a) A schematic of neutral competition experiments. A 50:50 mixture of wild type and labelled invader strain is inoculated without antibiotics into unoccupied chambers. (b) Labelled cells are sparsely distributed in a gaseous phase. (c) Steric interactions and proliferations produce band-like patterns in a jammed phase. The population dynamics are dominated by a small number of cells at the bottom of a cavity. (d) Diversity is rapidly lost in a jammed phase. A cluster of GFP-tagged cells is pushed out of the chamber by the population growth in a few generations.
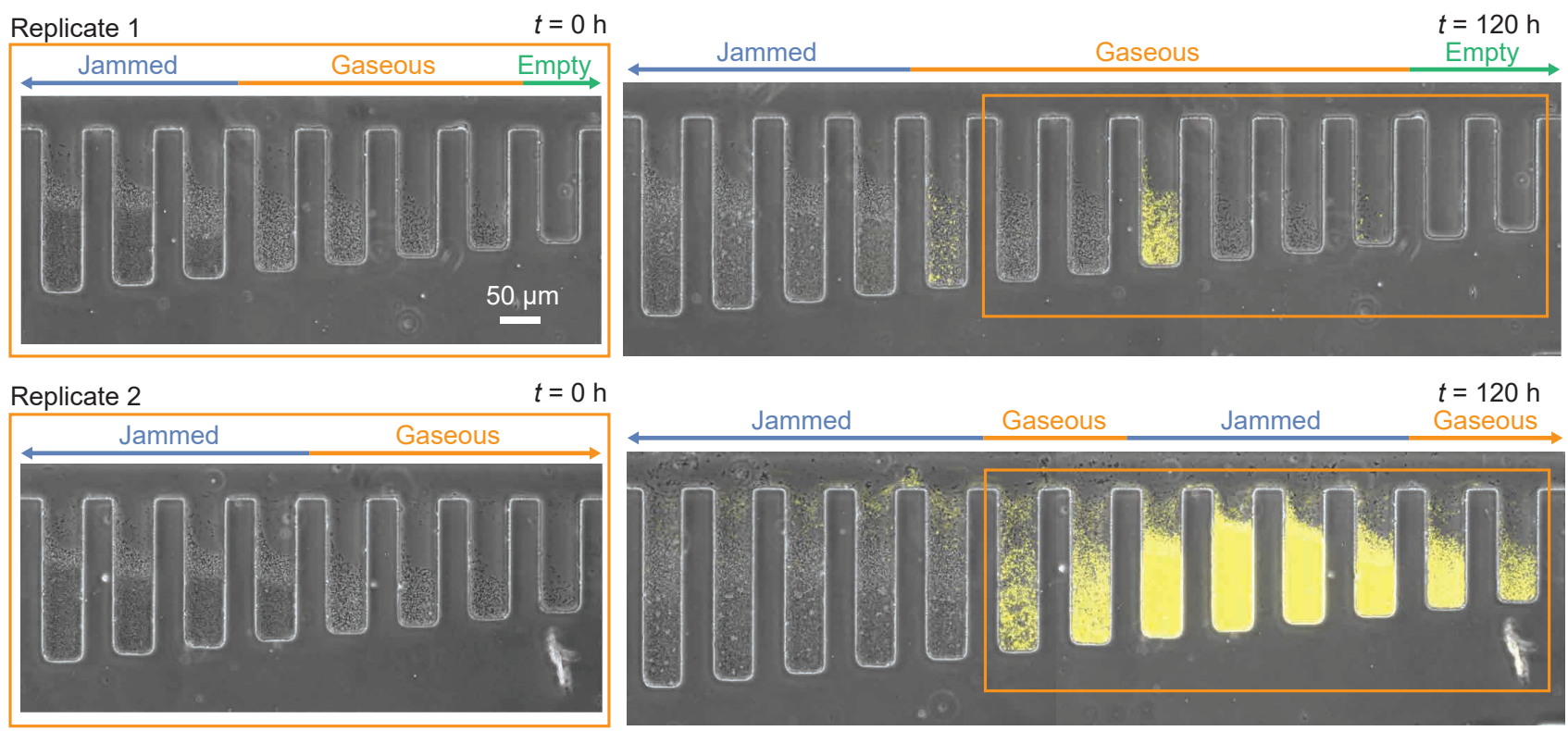

Figure ED7. Replicas of invasion experiments with $10 \mu \mathrm{g} / \mathrm{mL}$ tetracycline. Replicas from other rows on the same microfluidic chip. Orange frames show the same positions. Colonization resistance of the jammed phases was consistently observed, while the rate of invasion varied across replicas (less successful in the replica 1, and more successful in the replica 2). 
a) Acetobacter pasteurianus

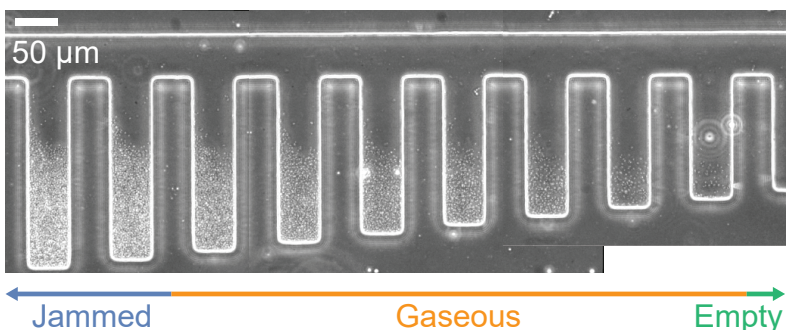

b) Acetobacter tropicalis

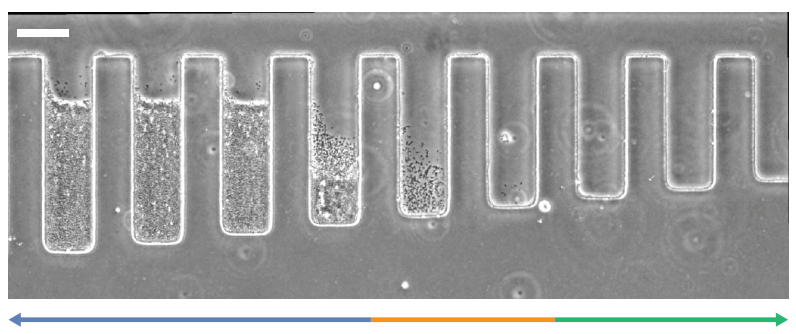

c) Lactococcus lactis

Day 5

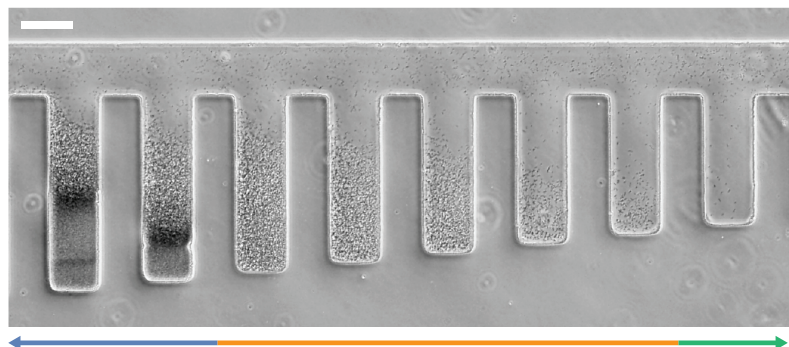

d) Vibrio cholerae

Day 2

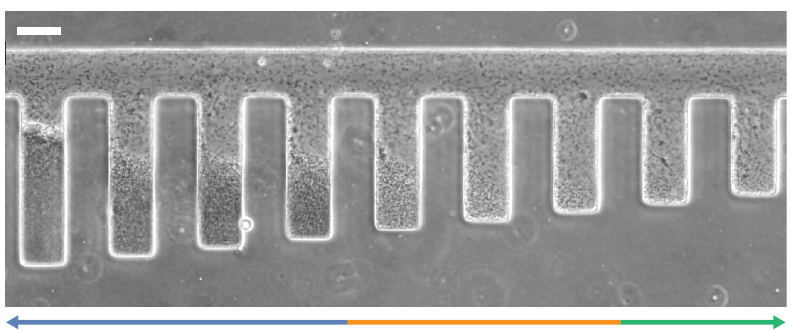

Figure ED8. Three colonization phases are observed in different bacterial species. Pictures were taken after $2 \sim 6$ days of incubation in microfluidic devices. We observed biofilm formation (b) and nutrient depletion (c), but qualitatively, they did not change the colonization patterns. Scale bars indicate $50 \mu \mathrm{m}$.
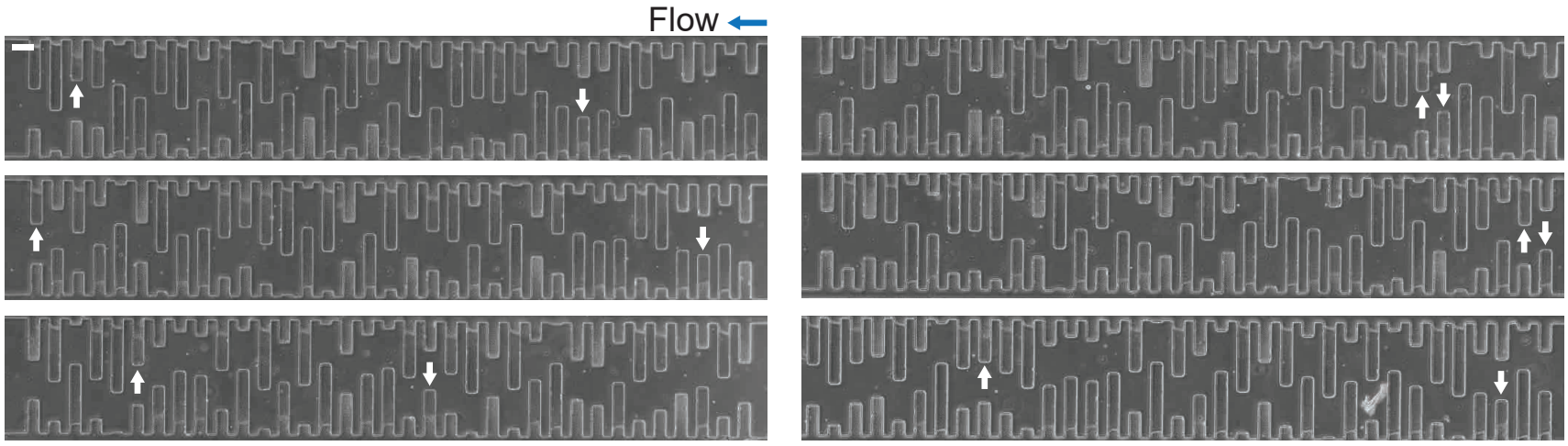

Figure ED9. Colonization patterns in randomized panflutes. The effect of anterior populations in the same row was tested by randomizing the order of chambers. The transition to a phase-separated state was observed independent of the order of the chambers. White arrows show the onset of jamming. (Scale bar indicates $100 \mu \mathrm{m}$ ). 